\title{
Manifold structures for sets of solutions of the general relativistic constraint equations
}

\author{
Piotr T. Chruściel* \\ Erwann Delay ${ }^{\dagger}$ \\ Erwin Schrödinger Institute \\ Vienna, Austria
}

\begin{abstract}
We construct manifold structures on various sets of solutions of the general relativistic initial data sets.
\end{abstract}

AMS 83C05, 58J99; PACS 04.20.Cv

\section{Introduction}

A natural question that arises in general relativity is whether some sets of solutions of the, say vacuum, constraint equations carry a manifold structure. For example, it is useful to have a Banach manifold structure on the set of asymptotically flat solutions of the constraint equations when trying to minimise the ADM mass $[7,8,10]$. Appropriate manifold structures allow one to use tools such as the Smale-Sard theorem, or the Baire category theorem, when discussing genericity of some properties of solutions of the Einstein equations. The existence of a Fréchet manifold structure ${ }^{1}$ for (a subset of) the set of solutions of the vacuum constraint equations on a compact manifold is a consequence of linearisation stability studies of Fischer, Marsden and Moncrief, (see [20,21] and references therein, compare Theorem 4.6 below). The results there have also been studied in the context of asymptotically flat initial data sets on a manifold with compact interior in [1].

While the above results might be satisfactory for several purposes, they do not lead to a Banach manifold of solutions. In finite dimension there is no

${ }^{*}$ Visiting Scientist. Permanent address: Département de Mathématiques, Faculté des Sciences, Parc de Grandmont, F37200 Tours, France. Partially supported by a Polish Research Committee grant 2 P03B 073 24, by the Erwin Schrödinger Institute, and by a travel grant from the Vienna City Council; email piotr@gargan.math.univ-tours.fr, URL www.phys. univ-tours.fr/ piotr

${ }^{\dagger}$ Visiting Scientist. Permanent address: Département de Mathématiques, Faculté des Sciences, rue Louis Pasteur, F84000 Avignon, France. Partially supported by the ACI program of the French Ministry of Research and by the Erwin Schrödinger Institute; email delay@gargan.math.univ-tours.fr

${ }^{1}$ As pointed out to us by R. Bartnik, the argument of [20] leads to a loss of regularity of the metric, forcing one to work with $C^{\infty}$ objects as far as the manifold structure is concerned. 
need to introduce a distinction between Banach, or Hilbert, or Fréchet manifold structure; however, the differences are significant in infinite dimension, because some facts which are true in Hilbert spaces are not necessarily true in all Banach spaces. Similarly some properties of Banach spaces do not carry over to Fréchet spaces. (The reader is referred to $[18,24,25,29]$ for analysis on infinite dimensional manifolds.)

In the asymptotically flat case an alternative method has been developed by R. Bartnik ${ }^{2}$ for constructing a Hilbert manifold structure on the space of solutions of the vacuum constraints, essentially based on the conformal method. It uses a (weighted) $H^{k} \times H^{k+1}$ topology, $k \geq 1$, on the space of $(K, g)$ 's, and it is clear that the method generalises to certain other settings of interest. Such spaces are well adapted to the evolution problem, at least for $k$ large enough. However, in that method one does not have the flexibility in controlling boundary or asymptotic behavior which is provided by the Corvino-Schoen $[12$, 15] version of the Fischer-Marsden-Moncrief approach.

The purpose of this note is to show that a Banach manifold structure can be obtained by a variation of the Fischer-Marsden-Moncrief-Corvino-Schoen method. It turns out that a direct application of the standard a priori estimates [28] for the construction below does not seem to lead to a manifold structure based on Sobolev spaces, which would have been more natural for the evolution problem, and which would have led to a Hilbert manifold structure. Instead, e.g. on compact manifolds without boundary, we obtain a manifold modeled on (a subset of) the space $C^{k, \alpha} \times C^{k, \alpha}, k \geq 4, \alpha \in(0,1)$ of symmetric tensors. This appears somewhat surprising at first sight, as a natural set-up for the evolution problem (regardless of the Sobolev vs Hölder space issue) might seem to be one where the differentiability of the extrinsic curvature tensor $K$ is one order less than that of the metric $g$. On the other hand, since $K$ 's can be thought of as variations of $g$ 's, from a manifold structure point of view it seems natural that the $K$ 's live in a space with the same differentiability as $g$. Whatever the natural space is, the $C^{k, \alpha} \times C^{k, \alpha}$ topology or weighted versions thereof are the ones which are obtained by the method here; this is a rather unexpected consequence of our analysis in this paper. As already pointed out, and as made clear in the applications below, the manifolds of initial data obtained here exhibit more structure than what is obtained by the conformal method and its variations.

In our construction of the manifold structure we use a smoothing device to recover the loss of regularity inherent to the Fischer-Marsden-Moncrief approach. This allows us to work consistently in spaces with finite differentiability, leading to the Banach manifold structure described above. We use a general approach of weighted spaces as in [12], which allows a simultaneous treatment of the compact case with or without boundary, and of the asymptotically flat case, and of the conformally compactifiable case, with families of different topologies.

All the results presented below remain valid in the time-symmetric setting, $K=0$. This implies that all the manifold structures presented here have their

\footnotetext{
${ }^{2}$ R. Bartnik, in preparation. Some similar ideas have also been considered by L. Andersson (private communication).
} 
obvious counterparts for the set of Riemannian metrics with prescribed scalar curvature.

This paper is organised as follows: In Section 2 we review the framework of [12], and we show how the results there can be used to define a local Hilbert manifold of solutions near a given solution; the resulting manifolds can not be patched together in general because of insufficient regularity of the operators involved. In Section 3 we present our basic regularisation procedure, which turns out to still be insufficient to provide a (global) Hilbert manifold structure. In Section 4 we therefore pass to an analysis in weighted Hölder spaces. We prove there that (the KID-free part of) the level sets of the constraints map are, globally, embedded submanifolds in a Banach space, see Theorem 5.2, under very general conditions on the weights; this is the main result of the paper. In fact, we prove that the level sets of the constraint map foliate (in a sense made precise in Remark 5.10 below) the KID-free part of the space of all $(K, g)$ 's. In Section 6 we show that the hypotheses made in Theorem 5.2 are fulfilled on compact manifolds with or without boundary, or on asymptotically compactifiable manifolds, or on asymptotically flat manifolds. In appendix A we prove a lemma which provides a submanifold structure in Banach spaces under rather general conditions, as well as a foliation result. In Appendix B we present two regularisation procedures in weighted spaces, as needed in applications of the submanifold Theorem 5.2. For the convenience of the reader those results from [12] which play a key role in the current construction have been presented in detail, including some introductory comments borrowed from [12] whenever useful for the clarity of the argument.

\section{The construction}

Let

$$
\mathscr{C}(K, g):=(J(K, g), \rho(K, g))
$$

be the general relativistic constraints map:

$$
\left(\begin{array}{l}
J \\
\rho
\end{array}\right)(K, g):=\left(\begin{array}{l}
2\left(-\nabla^{j} K_{i j}+\nabla_{i} \operatorname{tr} K\right) \\
R(g)-|K|^{2}+(\operatorname{tr} K)^{2}-2 \Lambda
\end{array}\right),
$$

where $\Lambda$ is the cosmological constant. (The function $c^{4} \rho / 16 \pi G$ is the energy density of the matter fields, while $c^{4} J / 16 \pi G$ is the energy-momentum flux vector.) The general relativistic constraint equations are $\mathscr{C}(K, g)=0$, whatever the space-dimension $n$. As those equations are trivial in space-dimension zero and one, in the remainder of this work we shall assume that $n \geq 2$.

Let $h=\delta g$ and $Q=\delta K$, the linearisation $P_{(K, g)}$ of the constraints map at 
$(K, g)$ reads

$$
P_{(K, g)}(Q, h)=\left(\begin{array}{c}
-K^{p q} \nabla_{i} h_{p q}+K^{q}{ }_{i}\left(2 \nabla^{j} h_{q j}-\nabla_{q} h_{l}\right) \\
-2 \nabla^{j} Q_{i j}+2 \nabla_{i} \operatorname{tr} Q-2\left(\nabla_{i} K^{p q}-\nabla^{q} K^{p}{ }_{i}\right) h_{p q} \\
-\Delta(\operatorname{tr} h)+\operatorname{div} \operatorname{div} h-\langle h, \operatorname{Ric}(g)\rangle+2 K^{p l} K^{q} h_{p q} \\
-2\langle K, Q\rangle+2 \operatorname{tr} K(-\langle h, K\rangle+\operatorname{tr} Q)
\end{array}\right) .
$$

Recall that a KID is defined as a solution $(N, Y)$ of the set of equations $P_{(K, g)}^{*}(Y, N)=0$, where $P_{(K, g)}^{*}$ is the formal adjoint of $P_{(K, g)}$ :

$$
P_{(K, g)}^{*}(Y, N)=\left(\begin{array}{l}
2\left(\nabla_{(i} Y_{j)}-\nabla^{l} Y_{l} g_{i j}-K_{i j} N+\operatorname{tr} K N g_{i j}\right) \\
\nabla^{l} Y_{l} K_{i j}-2 K^{l}{ }_{i} \nabla_{j)} Y_{l}+K^{q} \nabla_{q} Y^{l} g_{i j}-\Delta N g_{i j}+\nabla_{i} \nabla_{j} N \\
+\left(\nabla^{p} K_{l p} g_{i j}-\nabla_{l} K_{i j}\right) Y^{l}-N \operatorname{Ric}(g)_{i j}+2 N K_{i}^{l} K_{j l}-2 N(\operatorname{tr} K) K_{i j}
\end{array}\right) .
$$

We shall denote by $\mathscr{K}(\Omega)$ the set of KIDs defined on an open set $\Omega$. In vacuum space-times $(\mathscr{M}, g)$ KIDs on a spacelike hypersurface $\Omega$ are in one-to-one correspondence with Killing vectors of $g$ [27] on the domain of dependence of $\Omega$. A similar statement holds in electro-vacuum for appropriately invariant initial data for the electromagnetic field, the reader is referred to [9] for some comments about general matter fields.

Following [12], we will be using weighted function spaces defined as follows. Let $\phi$ and $\psi$ be two smooth strictly positive functions ${ }^{3}$ on $M$. For $k \in \mathbb{N}$ let $H_{\phi, \psi}^{k}(g)$ be the space of $H_{\text {loc }}^{k}$ functions or tensor fields such that the norm ${ }^{4}$

$$
\|u\|_{H_{\phi, \psi}^{k}(g)}:=\left(\int_{M}\left(\sum_{i=0}^{k} \phi^{2 i}\left|\nabla^{(i)} u\right|_{g}^{2}\right) \psi^{2} d \mu_{g}\right)^{\frac{1}{2}}
$$

is finite, where $\nabla^{(i)}$ stands for the tensor $\underbrace{\nabla \ldots \nabla}_{i \text { times }} u$, with $\nabla-$ the Levi-Civita covariant derivative of $g$; we assume throughout that the metric is at least $W_{\text {loc }}^{1, \infty}$; higher differentiability will be usually indicated whenever needed. For $k \in \mathbb{N}$ we denote by $\stackrel{\circ}{H}_{\phi, \psi}^{k}$ the closure in $H_{\phi, \psi}^{k}$ of the space of $H^{k}$ functions or tensors which are compactly (up to a negligible set) supported in $M$, with the norm induced from $H_{\phi, \psi}^{k}$. The $\stackrel{\circ}{H}_{\phi, \psi}^{k}$ 's are Hilbert spaces with the obvious scalar product associated to the norm (2.4). We will also use the following notation

$$
\stackrel{\circ}{H}^{k}:=\stackrel{\circ}{H}_{1,1}^{k}, \quad L_{\psi}^{2}:=\stackrel{\circ}{H}_{1, \psi}^{0}=H_{1, \psi}^{0},
$$

\footnotetext{
${ }^{3}$ We use the analysts' convention that a manifold $M$ is always open; thus a manifold $M$ with non-empty boundary $\partial M$ does not contain its boundary; instead, $\bar{M}:=M \cup \partial M$ is a manifold with boundary in the differential geometric sense. Unless explicitly specified otherwise no conditions on $M$ are made - e.g. that $\partial M$, if non-empty, is compact - except that $M$ is a smooth manifold; similarly no conditions e.g. on completeness of $(M, g)$, or on its radius of injectivity, are made.

${ }^{4}$ The reader is referred to $[4,5,23]$ for a discussion of Sobolev spaces on Riemannian manifolds.
} 
so that $L^{2} \equiv \stackrel{\circ}{H}^{0}:=\stackrel{\circ}{H}_{1,1}^{0}$. We set

$$
W_{\phi}^{k, \infty}:=\left\{u \in W_{\text {loc }}^{k, \infty} \text { such that } \phi^{i}\left|\nabla^{(i)} u\right|_{g} \in L^{\infty}\right\}
$$

with the obvious norm, and with $\nabla^{(i)} u$ - the distributional derivatives of $u$.

For $\phi$ and $\varphi-$ smooth strictly positive functions on $\mathrm{M}$, and for $k \in \mathbb{N}$ and $\alpha \in[0,1]$, we define $C_{\phi, \varphi}^{k, \alpha}$ the space of $C^{k, \alpha}$ functions or tensor fields for which the norm

$$
\begin{aligned}
\|u\|_{C_{\phi, \varphi}^{k, \alpha}(g)}=\sup _{x \in M} \sum_{i=0}^{k}\left(\left\|\varphi \phi^{i} \nabla^{(i)} u(x)\right\|_{g}\right. \\
\left.+\sup _{0 \neq d_{g}(x, y) \leq \phi(x) / 2} \varphi(x) \phi^{i+\alpha}(x) \frac{\left\|\nabla^{(i)} u(x)-\nabla^{(i)} u(y)\right\|_{g}}{d_{g}^{\alpha}(x, y)}\right)
\end{aligned}
$$

is finite.

We will only consider weight functions with the property that there exists $\ell \in \mathbb{N} \cup\{\infty\}$ such that ${ }^{5}$ for $0 \leq i<\ell$ we have

$$
\left|\phi^{i-1} \nabla^{(i)} \phi\right|_{g} \leq C_{i}, \quad\left|\phi^{i} \psi^{-1} \nabla^{(i)} \psi\right|_{g} \leq C_{i}, \quad\left|\phi^{i} \varphi^{-1} \nabla^{(i)} \varphi\right|_{g} \leq C_{i},
$$

for some constants $C_{i}$. The following situations seem to be of main interest:

- If $M$ is compact without boundary we will use $\phi=\psi=\varphi=1$.

- If $\partial M$ is compact, smooth, and non-empty, we will use for $\phi=x$ a function which is a defining function for the boundary, at least in a neighborhood of the boundary; that is, any smooth non-negative function on $\bar{M}$ such that $\partial M$ is precisely the zero-level set of $x$, with $d x$ without zeros on $\partial M$. Then $\psi$ and $\varphi$ will be a power of $x$ on a neighborhood of $\partial M$. Condition (2.5) will hold for metrics which are smooth up-to-boundary near $\partial M$.

- If $M$ contains an asymptotically flat region, $\phi$ will behave as $r$, while $\varphi$ and $\psi$ will behave as a power of $r$ in the asymptotically flat region; (2.5) will hold for a large class of asymptotically flat metrics.

- If $M$ contains a conformally compactifiable region, then in a neighborhood of the conformal boundary $\phi$ will be taken to be 1 , while $\psi$ will be a power of the defining function of the conformal boundary.

- Exponentially weighted versions of the above will also be considered.

In all those situations one can obtain elliptic estimates in weighted spaces for the equations considered here by covering and scaling arguments together with the standard interior elliptic estimates on compact sets (cf., e.g. $[2,3,6,11,22,26])$. We will refer to this as the scaling property. More precisely, we shall say that the scaling property holds (with respect to some weighted Sobolev spaces with weight functions $\psi$ and $\phi$, and/or weighted Hölder spaces with weight functions $\varphi$ and $\phi$, whichever ones are being used will always be obvious from the context)

\footnotetext{
${ }^{5}$ Conditions (2.5) will typically impose $\ell$ restrictions on the behavior of the metric and its derivatives in the asymptotic regions; it is therefore essential to allow $\ell<\infty$ if one does not wish to impose an infinite number of such conditions.
} 
if there exists a covering of $M$ by a family of sets $\Omega_{\alpha}$, for $\alpha$ in some index set $I$, together with scaling transformations $\phi_{\alpha}: \Omega_{\alpha} \rightarrow \hat{\Omega}_{\alpha}$ on each of the sets $\Omega_{\alpha}$, such that the transformed fields $\left(\hat{K}_{\alpha}, \hat{g}_{\alpha}\right)$ on $\Omega_{\alpha}$ are in ${ }^{6}$ in $W^{3, \infty}\left(\hat{\Omega}_{\alpha}\right) \times W^{4, \infty}\left(\hat{\Omega}_{\alpha}\right)$, and such that the usual interior elliptic estimates on the $\hat{\Omega}_{\alpha}$ 's can be pieced together to a weighted estimate, such as (4.7), for the original fields. Some sufficient conditions for the scaling property are discussed in [12, Appendix B]. We note that the scaling transformation of the fields on $\hat{\Omega}_{\alpha},(K, g) \rightarrow\left(\hat{K}_{\alpha}, \hat{g}_{\alpha}\right)$, will typically consist of a pull-back of the fields, accompanied perhaps by a constant conformal rescaling. The "scaling property" is a condition both on the metric $g$, the extrinsic curvature tensor $K$, and on the weight functions involved: indeed, both the metric coefficients, the connection coefficients, as well as their derivatives, etc., which appear in our equations must have appropriate behavior under the above transformations so that the required estimates can be established.

Conditions (2.5) guarantee the following:

Lemma 2.1 Let $k \in \mathbb{Z}, k \geq-2$. Suppose that $t^{7} g \in W_{\text {loc }}^{k+3, \infty}$ and that

$$
\begin{gathered}
\operatorname{Ric}(g) \in \phi^{-2} W_{\phi}^{k+2, \infty}(g), \\
K \in \phi^{-1} W_{\phi}^{k+3, \infty}(g) .
\end{gathered}
$$

If (2.5) holds with $0 \leq i \leq k+2$, then the linear operators

$$
\begin{aligned}
& P_{(K, g)}^{*}: \stackrel{\circ}{H}_{\phi, \psi}^{k+3}(g) \times \stackrel{\circ}{H}_{\phi, \psi}^{k+4}(g) \longrightarrow \phi^{-1} \stackrel{\circ}{H}_{\phi, \psi}^{k+2}(g) \times \phi^{-2} \stackrel{\circ}{H}_{\phi, \psi}^{k+2}(g), \quad k \geq-2, \\
& P_{(K, g)}: \psi^{2}\left(\phi \stackrel{\circ}{H}_{\phi, \psi}^{k+2}(g) \times \phi^{2} \stackrel{\circ}{H}_{\phi, \psi}^{k+2}(g)\right) \longrightarrow \psi^{2}\left(\stackrel{\circ}{H}_{\phi, \psi}^{k+1}(g) \times \stackrel{\circ}{H}_{\phi, \psi}^{k}(g)\right), \quad k \geq 0,
\end{aligned}
$$

are well defined, and bounded.

The following operator is of interest in our context,

$$
L_{\phi, \psi}:=\psi^{-2} P_{(K, g)} \Phi \psi^{2} \Phi P_{(K, g)}^{*},
$$

where $\Phi$ is defined by

$$
\Phi(x, y):=\left(\phi x, \phi^{2} y\right) .
$$

A useful inequality to make things work is the following:

$$
C\left\|\Phi P_{\left(K_{0}, g_{0}\right)}^{*}(Y, N)\right\|_{L_{\psi}^{2}\left(g_{0}\right)} \geq\|Y\|_{L_{\psi}^{2}\left(g_{0}\right)}+\|N\|_{\stackrel{\circ}{\phi}, \psi_{1}^{1}\left(g_{0}\right)} \cdot
$$

Let $\mathcal{K}_{0}$ be kernel of

$$
\Phi P_{\left(K_{0}, g_{0}\right)}^{*}: \stackrel{\circ}{H}_{\phi, \psi}^{1}\left(g_{0}\right) \times \stackrel{\circ}{H}_{\phi, \psi}^{2}\left(g_{0}\right) \longrightarrow L_{\psi}^{2}\left(g_{0}\right) \times L_{\psi}^{2}\left(g_{0}\right),
$$

\footnotetext{
${ }^{6}$ It is conceivable that in some situations less a priori regularity on the $\left(\hat{K}_{\alpha}, \hat{g}_{\alpha}\right)$ 's can be assumed, but this is the setup which seems to play the most important role in our paper; the reader should be able to adapt the differentiability conditions to his needs if required.

${ }^{7}$ The local differentiability conditions follow from the requirement that the $k+$ fourth covariant derivatives of $N$ and the $k+$ third ones of $Y$ can be defined in a distributional sense; both of those conditions are fulfilled by a metric $g \in W_{\text {loc }}^{k+3, \infty}$ - the reader should note that the first covariant derivatives of $N$ do not involve the Christoffel symbols of $g$ since $N$ is a function.
} 
and let $\mathcal{K}_{0}{ }^{\perp_{g_{0}}}$ be its $L_{\psi}^{2}\left(g_{0}\right) \oplus L_{\psi}^{2}\left(g_{0}\right)$-orthogonal. We denote by $\pi_{\mathcal{K}_{0}^{\perp g}}$ the $L_{\psi}^{2}(g)$ projection onto $\mathcal{K}_{0}^{\perp_{g}}$. The following result, $\operatorname{proved}^{8}$ in [12], is a weighted equivalent of those in $[13,14,16]$ (compare [15]):

TheOREM 2.2 ( $\left[12\right.$, Theorem 3.6]) Let $k \geq 0, g_{0} \in W_{\text {loc }}^{k+4, \infty}$, suppose that $(2.5)$ holds with $0 \leq i \leq 4+k$, that

$$
\operatorname{Ric}\left(g_{0}\right) \in \phi^{-2} W_{\phi}^{k+2, \infty}\left(g_{0}\right), \quad K_{0} \in \phi^{-1} W_{\phi}^{k+3, \infty}\left(g_{0}\right),
$$

and that the weights $\phi$ and $\psi$ have the scaling property. If there exists a compact set $\mathscr{K} \subset M$ such that for all $\stackrel{\circ}{H}_{\phi, \psi}^{1}\left(g_{0}\right)$ vector fields $Y$ and $\stackrel{\circ}{H}_{\phi, \psi}^{2}\left(g_{0}\right)$ functions $N$, both supported in $M \backslash \mathscr{K}$, the inequality (2.12) holds, then for all $(K, g)$ close to $\left(K_{0}, g_{0}\right)$ in $\phi^{-1} W_{\phi}^{k+3, \infty}\left(g_{0}\right) \times W_{\phi}^{k+4, \infty}\left(g_{0}\right)$ norm, the map

$$
\pi_{\mathcal{K}_{0}^{\perp g}} L_{\phi, \psi}: \mathcal{K}_{0}^{\perp_{g}} \cap\left(\stackrel{\circ}{H}_{\phi, \psi}^{k+3}(g) \times \stackrel{\circ}{H}_{\phi, \psi}^{k+4}(g)\right) \longrightarrow \mathcal{K}_{0}^{\perp_{g}} \cap\left(\stackrel{\circ}{H}_{\phi, \psi}^{k+1}(g) \times \stackrel{\circ}{H}_{\phi, \psi}^{k}(g)\right)
$$

is an isomorphism such that the norm of its inverse is bounded independently of $(K, g)$.

We will see how to use this result, and certain variations thereof, to obtain a manifold structure on various sets of solutions of the vacuum constraint equations. More generally, one obtains a manifold structure on the set of initial data with $(J, \rho)$-fixed. Recall that a Banach manifold is a Hausdorff topological space $M$ such that for every $p \in M$ there exists a neighborhood $\mathscr{U}_{p} \subset M$ of $p$ equipped with a homeomorphism $\phi_{p}$ from $\mathscr{U}_{p}$ to an open subset $\mathscr{O}_{p}$ of some Banach space $B_{p}$. The couple $\left(\mathscr{U}_{p}, \phi_{p}\right)$, and sometimes simply the set $\mathscr{U}_{p}$, will be called a coordinate patch. On overlapping coordinate patches $\mathscr{U}_{p}$ and $\mathscr{U}_{q}$ the maps $\phi_{p} \circ \phi_{q}^{-1}$ are supposed to be smooth diffeomorphisms from their domains $\phi_{q}\left(\mathscr{U}_{p} \cap \mathscr{U}_{q}\right) \subset B_{q}$ to their images $\phi_{p}\left(\mathscr{U}_{p} \cap \mathscr{U}_{q}\right) \subset B_{p}$. One can similarly define the notion of a Hilbert manifold, and of a Fréchet manifold.

A connected embedded submanifold of an open subset of a Banach space is always a manifold modeled on any of its tangent spaces (which are all necessarily diffeomorphic, compare Corollary 5.11 below). We will actually prove that the level sets of the constraint map form embedded submanifolds in such a space, which will provide the desired manifold structure.

We start with the following observation:

Proposition 2.3 (Local Hilbert manifold (and submanifold)) Under the hypotheses of Theorem 2.2, suppose that there are no KIDs:

$$
\stackrel{\circ}{H}_{\phi, \psi}^{k+3}\left(g_{0}\right) \times \stackrel{\circ}{H}_{\phi, \psi}^{k+4}\left(g_{0}\right) \supset \mathcal{K}_{0}=\{0\} .
$$

\footnotetext{
${ }^{8}$ Actually in [12] the hypothesis is made that the cosmological constant $\Lambda$ vanishes, which is not assumed here (compare (2.1)). The inclusion of a cosmological constant does not require any modifications of the proofs there, insofar as the results discussed here are concerned.
} 
Assume that the map

$$
\begin{gathered}
\psi^{2}\left(\phi \stackrel{\circ}{H}_{\phi, \psi}^{k+2}\left(g_{0}\right) \times \phi^{2} \stackrel{\circ}{H}_{\phi, \psi}^{k+2}\left(g_{0}\right)\right) \longrightarrow \stackrel{\circ}{H}_{\phi, \psi}^{k+1}\left(\left(g_{0}\right)\right) \times \stackrel{\circ}{H}_{\phi, \psi}^{k}\left(\left(g_{0}\right)\right) \\
(\delta K, \delta g) \longmapsto \psi^{-2}\left\{\left(\begin{array}{c}
J \\
\rho
\end{array}\right)\left[\left(K_{0}, g_{0}\right)+(\delta K, \delta g)\right]-\left(\begin{array}{c}
J \\
\rho
\end{array}\right)\left(K_{0}, g_{0}\right)\right\}
\end{gathered}
$$

is differentiable at zero. Then the set

$$
\begin{aligned}
\mathcal{S}_{0}=\left\{(Q, h) \in \psi^{2}\left(\phi \stackrel{\circ}{H}_{\phi, \psi}^{k+2}\left(g_{0}\right) \times \phi^{2} \stackrel{\circ}{H}_{\phi, \psi}^{k+2}\left(g_{0}\right)\right),\right. & \\
& \left.(J, \rho)\left(K_{0}+Q, g_{0}+h\right)-(J, \rho)\left(K_{0}, g_{0}\right)=0\right\}
\end{aligned}
$$

is an embedded submanifold of $\psi^{2}\left(\phi \stackrel{\circ}{H}_{\phi, \psi}^{k+2}\left(g_{0}\right) \times \phi^{2} \stackrel{\circ}{H}_{\phi, \psi}^{k+2}\left(g_{0}\right)\right)$ in a neighborhood of zero.

Proof: A rather general justification is provided by Lemma A.1, Appendix A, by setting $u=D u(0)=\psi^{2} \Phi^{2} P_{\left(K_{0}, g_{0}\right)}^{*}, v=\psi^{-2}\left[(J, \rho)\left(K_{0}+., g_{0}+.\right)-\right.$ $\left.(J, \rho)\left(K_{0}, g_{0}\right)\right], \quad D v(0)=\psi^{-2} P_{\left(K_{0}, g_{0}\right)}, \quad E=\stackrel{\circ}{H}_{\phi, \psi}^{k+3}\left(g_{0}\right) \times \stackrel{\circ}{H}_{\phi, \psi}^{k+4}\left(g_{0}\right), \quad F=$ $\psi^{2} \Phi\left(\stackrel{\circ}{H}_{\phi, \psi}^{k+2}\left(g_{0}\right) \times \stackrel{\circ}{H}_{\phi, \psi}^{k+2}\left(g_{0}\right)\right)$, and $G=\stackrel{\circ}{H}_{\phi, \psi}^{k+1}\left(g_{0}\right) \times \stackrel{\circ}{H}_{\phi, \psi}^{k}\left(g_{0}\right)$. The linear map $u$ is continuous by Lemma (2.1), hence differentiable, while $v$ is differentiable by hypothesis. The required isomorphism property for $D v(0) \circ D u(0)$ follows from Theorem 2.2.

ExAMPLE 2.4 To motivate and illustrate the results so far, consider the case of a compact manifold $M$ without boundary; other examples of interest will be treated in detail in Section 6. We choose $\phi=\psi \equiv 1$, so the spaces involved are standard Sobolev spaces. As $M$ is compact we can take $\mathscr{K}=M$ so that condition (2.12) is trivially satisfied. The smoothness hypotheses on the map (3.2) are satisfied by standard calculus in Sobolev spaces. If $\left(K_{0}, g_{0}\right)$ is a $C^{k+4} \times$ $C^{k+3}$ initial data set without KIDs, Proposition 2.3 provides a manifold of $H^{k+2} \times H^{k+2}$ solutions of the constraint equations passing through $\left(K_{0}, g_{0}\right)$.

Example 2.4 clearly exhibits an unfortunate differentiability mismatch here which leads to an essential obstruction when trying to glue together the coordinate patches obtained so far, and which therefore prevents one from obtaining a Hilbert manifold structure on the set of all solutions of the constraints equations using the method above. In Section 5 we will show that a (different) manifold structure can nevertheless be obtained using the following approach: consider a data set $\left(K_{1}, g_{1}\right)$ without KIDs in an appropriate Hölder differentiability class, let $\left(\delta K_{1}, \delta g_{1}\right)$ be a solution of the constraints with the same differentiability class, and suppose that you can smooth out $\left(K_{1}+\delta K_{1}, g_{1}+\delta g_{1}\right)$ to a smooth set $(K, g)$, in a way consistent with the set-up of Theorem 3.1. If one can solve the equation

$\left(\begin{array}{c}J \\ \rho\end{array}\right)\left(\left(K_{0}+\delta K, g_{0}+\delta g\right)+\psi^{2} \Phi^{2} P_{(K, g)}^{*}(Y, N)\right)-\left(\begin{array}{c}J \\ \rho\end{array}\right)\left(K_{0}+\delta K, g_{0}+\delta g\right)=\left(\begin{array}{c}\delta J \\ \delta \rho\end{array}\right)$,

then one has a better chance of ending in a space with the original differentiability. 


\section{A regularised problem}

The aim of this section is to implement the above in weighted Sobolev spaces. Consider again a compact manifold without boundary, let $(K, g)$ be of $C^{k+2, \alpha} \times$ $C^{k+2, \alpha}$ differentiability class, and first regularise $(K, g)$ by the usual convolution method to obtain a new smooth couple $\left(K_{\varepsilon}, g_{\varepsilon}\right)$, then define

$$
P_{\varepsilon}^{*}:=P_{\left(K_{\varepsilon}, g_{\varepsilon}\right)}^{*}
$$

Roughly speaking, the equation we will attempt to solve will be (2.16) with $P_{(K, g)}^{*}$ there replaced by $P_{\varepsilon}^{*}$. The idea is to solve that equation for $(Y, N) \in$ $C^{3, \alpha} \times C^{4, \alpha}$, and then use elliptic regularity to get to $(Y, N) \in C^{k+3, \alpha} \times C^{k+4, \alpha}$, obtaining the desired extrinsic curvature and metric in $C^{k+2, \alpha} \times C^{k+2, \alpha}$; we emphasise that one would not get that last differentiability without the regularisation. Somewhat more generally, let $0 \leq k_{0} \leq k-2$, still in the compact boundaryless case one has the following convergence property:

$$
P_{\varepsilon}^{*} \rightarrow P^{*} \text { in } L\left(\stackrel{\circ}{H}_{\phi, \psi}^{k_{0}+3}\left(g_{0}\right) \times \stackrel{\circ}{H}_{\phi, \psi}^{k_{0}+4}\left(g_{0}\right), \phi^{-1} \stackrel{\circ}{H}_{\phi, \psi}^{k_{0}+2}\left(g_{0}\right) \times \phi^{-2} \stackrel{\circ}{H}_{\phi, \psi}^{k_{0}+2}\left(g_{0}\right)\right),
$$

which is precisely what is needed to make the arguments work. In general we will therefore assume that we have a smoothing operation $(K, g) \rightarrow\left(K_{\varepsilon}, g_{\varepsilon}\right)$ such that

$$
\left(K_{\varepsilon}, g_{\varepsilon}\right) \rightarrow_{\varepsilon \rightarrow 0}(K, g) \text { in } \phi^{-1} W_{\phi}^{k_{0}+3, \infty}\left(g_{0}\right) \times W_{\phi}^{k_{0}+4, \infty}\left(g_{0}\right) .
$$

(In Appendix B we give conditions on the weight functions which guarantee that the smoothing operation with the above properties exists.) This leads to the following variation of Theorem 3.9 of [12]:

TheOREM 3.1 Let $k \geq 0, g_{0} \in W_{\text {loc }}^{k+4, \infty}$, assume that (3.1) holds with $k_{0}=k$ and suppose that (2.5) holds with $0 \leq i \leq 4+k$. Assume moreover that

$$
\operatorname{Ric}\left(g_{0}\right) \in \phi^{-2} W_{\phi}^{k+2, \infty}\left(g_{0}\right), \quad K_{0} \in \phi^{-1} W_{\phi}^{k+3, \infty}\left(g_{0}\right),
$$

and that the weights $\phi$ and $\psi$ have the scaling property. Suppose further that there exists a compact set $\mathscr{K} \subset M$ such that for all $\stackrel{\circ}{\phi}_{\phi, \psi}^{1}\left(g_{0}\right)$ vector fields $Y$ and $\stackrel{\circ}{H}_{\phi, \psi}^{2}\left(g_{0}\right)$ functions $N$, both supported in $M \backslash \mathscr{K}$, the inequality (2.12) holds. If the weights are such that the map

$$
\begin{gathered}
\mathcal{K}_{0}^{\perp g} \cap\left(\stackrel{\circ}{H}_{\phi, \psi}^{k+3}(g) \times \stackrel{\circ}{H}_{\phi, \psi}^{k+4}(g)\right) \longrightarrow \mathcal{K}_{0}^{\perp g} \cap\left(\stackrel{\circ}{H}_{\phi, \psi}^{k+1}(g) \times \stackrel{\circ}{H}_{\phi, \psi}^{k}(g)\right) \\
(Y, N) \longmapsto \pi_{\mathcal{K}_{0}^{\perp g}} \psi^{-2}\left\{\left(\begin{array}{c}
J \\
\rho
\end{array}\right)\left[(K, g)+\psi^{2} \Phi^{2} P_{\varepsilon}^{*}(Y, N)\right]-\left(\begin{array}{c}
\stackrel{J}{\rho}
\end{array}\right)(K, g)\right\}
\end{gathered}
$$

is differentiable in a neighborhood of zero, then it is bijective in a (perhaps smaller) neighborhood of zero, for all $\varepsilon$ small enough. In other words, for sufficiently small $\varepsilon$, there exists $\delta>0$ such that for all $(K, g)$ close to $\left(K_{0}, g_{0}\right)$ in $\phi^{-1} W_{\phi}^{k+3, \infty}\left(g_{0}\right) \times W_{\phi}^{k+4, \infty}\left(g_{0}\right)$, and for all pairs $(\delta J, \delta \rho) \in \psi^{2}\left(\stackrel{\circ}{H}_{\phi, \psi}^{k+1}(g) \times\right.$ $\left.\stackrel{\circ}{H}_{\phi, \psi}^{k}(g)\right)$ with norm less than $\delta$, there exists a solution

$$
(\delta K, \delta g)=\Phi \psi^{2} \Phi P_{\varepsilon}^{*}(Y, N) \in \psi^{2}\left(\phi H_{\phi, \psi}^{k+2}(g) \times \phi^{2} H_{\phi, \psi}^{k+2}(g)\right),
$$


close to zero, of the equation

$$
\pi_{\mathcal{K}_{0}^{\perp g}} \psi^{-2}\left\{\left(\begin{array}{c}
J \\
\rho
\end{array}\right)(K+\delta K, g+\delta g)-\left(\begin{array}{c}
J \\
\rho
\end{array}\right)(K, g)\right\}=\pi_{\mathcal{K}_{0}^{\perp_{g}}} \psi^{-2}\left(\begin{array}{c}
\delta J \\
\delta \rho
\end{array}\right) .
$$

The solutions of the form (3.3) with sufficiently small norm are unique.

PROOF: Instead of (3.4) we consider the projection of the equation

$$
\left(\begin{array}{c}
J \\
\rho
\end{array}\right)\left((K, g)+\psi^{2} \Phi^{2} P_{\varepsilon}^{*}(Y, N)\right)-\left(\begin{array}{c}
J \\
\rho
\end{array}\right)(K, g)=\left(\begin{array}{c}
\delta J \\
\delta \rho
\end{array}\right)
$$

If $\varepsilon$ is small enough then $\left(K_{\varepsilon}, g_{\varepsilon}\right)$ is close to $(K, g)$ in $\phi^{-1} W_{\phi}^{k_{0}+3, \infty}\left(g_{0}\right) \times$ $W_{\phi}^{k_{0}+4, \infty}\left(g_{0}\right)$ thus close to $\left(K_{0}, g_{0}\right)$ in the same space. Because of the high differentiability threshold assumed all the coefficients in the equations are in $L_{\text {loc }}^{\infty}$, and it is easy to check that the operator $P_{\varepsilon}^{*}$ converges to $P^{*}$ when $\varepsilon$ goes to zero in such a way that the estimates in Appendix G of [12] remain uniform. It follows that for $\varepsilon$ small enough $P_{\varepsilon}^{*}$ can be used in place of $P^{*}$ to define the right inverse needed in the arguments of Appendix G of [12].

One would like to use elliptic estimate arguments to show that if $(K, g)$ is smooth, and if $(\delta J, \delta \rho)$ is smooth, then the solution is smooth. We have not been able to implement such an argument in the spaces used above because of poor differentiability of the coefficients of the equations. This has the effect that the size of the neighborhood for which the theorem applies might depend upon $k$. This problem will be sidetracked by working in weighted Hölder spaces.

\section{Analysis in weighted Hölder spaces}

Before passing to an analysis of the regularised equation (2.16), let us show that the results established in [12] can be used to obtain a Fréchet manifold of smooth solutions of constraint equations without KIDs.

In order to obtain a coherent set-up in weighted Hölder spaces we will need to impose some more conditions on the weight functions $\phi, \varphi$, and $\psi$ :

1. First, note that $(2.5)$ can be rewritten as $\phi \in C_{\phi, \phi^{-1}}^{\ell-1}, \psi \in C_{\phi, \psi^{-1}}^{\ell-1}, \varphi \in$ $C_{\phi, \varphi^{-1}}^{\ell-1}$. When dealing with Hölder spaces one also needs to assume Hölder continuity of the derivative weights, so (renaming $\ell-1$ to $\ell$ ) we will assume:

$$
\phi \in C_{\phi, \phi^{-1}}^{\ell, \alpha}, \quad \psi \in C_{\phi, \psi^{-1}}^{\ell, \alpha}, \quad \varphi \in C_{\phi, \varphi^{-1}}^{\ell, \alpha} .
$$

2. As discussed in [12, Appendix B], the following conditions are useful for deriving the scaling property: Let us denote by $B_{p}$ the open ball of center $p$ with radius $\phi(p) / 2$. We assume that there exist constants $C_{1}, C_{2}, C_{3}>0$ such that for all $p \in M$ and all $y \in B_{p}$, we have

$$
\begin{aligned}
& C_{1}^{-1} \phi(p) \leq \phi(y) \leq C_{1} \phi(p), \\
& C_{2}^{-1} \varphi(p) \leq \varphi(y) \leq C_{2} \varphi(p), \\
& C_{3}^{-1} \psi(p) \leq \psi(y) \leq C_{3} \psi(p) .
\end{aligned}
$$


3. Since the tool to handle non-linearities in this paper is the inverse function theorem, we need to make sure that the changes in the initial data are small as compared to the data themselves. A necessary condition for that is that the new metric be uniformly equivalent to the original one. One way of ensuring this is

$$
\psi^{2} \phi^{2} C_{\phi, \varphi}^{k, \alpha}\left(g_{0}\right) \subset C_{\phi, 1}^{k, \alpha}\left(g_{0}\right) .
$$

This will hold under the following condition:

Proposition 4.1 The inequality

$$
\psi^{2} \phi^{2} \varphi^{-1} \leq C
$$

implies (4.5).

In order to check this the reader might wish to prove first that the conditions imposed so far imply that

Lemma 4.2 If $u \in C_{\phi, \varphi_{1}}^{k, \alpha}(g)$ and $v \in C_{\phi, \varphi_{2}}^{k, \alpha}(g)$, with one of the $\varphi_{a}$ 's satisfying (4.3) and $\phi$ satisfying (4.1) with $\ell \geq k$, then $u v \in C_{\phi, \varphi_{1} \varphi_{2}}^{k, \alpha}(g)$.

Lemma 4.2 can be used to show an equivalent of Lemma 2.1 in weighted Hölder spaces.

Clearly all those conditions are fulfilled when $\phi=\varphi=\psi=1$; they will also be fulfilled in the other examples of interest discussed in Section 6 .

To proceed further some terminology will be needed:

Definition 4.3 We will say that an operator $L$ from $\stackrel{\circ}{H}_{\phi, \psi}^{3} \times \stackrel{\circ}{H}_{\phi, \psi}^{4}$ to $\stackrel{\circ}{H}_{\phi, \psi}^{1} \times \stackrel{\circ}{H}_{\phi, \psi}^{0}$ satisfies the weighted elliptic regularity condition if there exists a constant $C$ such that for all $(Y, N)$ in $\stackrel{\circ}{H}_{\phi, \psi}^{3} \times \stackrel{\circ}{H}_{\phi, \psi}^{4}$ satisfying $L(Y, N) \in C_{\phi, \varphi}^{k+1, \alpha} \times C_{\phi, \varphi}^{k, \alpha}$ we have $(Y, N) \in C_{\phi, \varphi}^{k+3, \alpha} \times C_{\phi, \varphi}^{k+4, \alpha}$ with

$$
\|(Y, N)\|_{C_{\phi, \varphi}^{k+3, \alpha} \times C_{\phi, \varphi}^{k+4, \alpha}} \leq C\left(\|L(Y, N)\|_{C_{\phi, \varphi}^{k+1, \alpha} \times C_{\phi, \varphi}^{k, \alpha}}+\|(Y, N)\|_{H_{\phi, \psi}^{3} \times H_{\phi, \psi}^{4}}\right) .
$$

When $L_{\phi, \psi}$ defined in (2.10) satisfies the weighted elliptic regularity condition one has the following:

Proposition 4.4 (Proposition 3.16 of [12]) Let $k \in \mathbb{N}, 0<\alpha<1$, assume that (4.1) with $\ell \geq k+4$ holds, and that (4.2)-(4.4) and (4.6) hold. In addition to the hypotheses of Theorem 3.1 with $\varepsilon=0$, suppose that $g_{0} \in C^{k+4, \alpha}$, and that

$$
\operatorname{Ric}\left(g_{0}\right) \in \phi^{-2} C_{\phi, 1}^{k+2, \alpha}\left(g_{0}\right), \quad K_{0} \in \phi^{-1} C_{\phi, 1}^{k+3, \alpha}\left(g_{0}\right) .
$$

We further assume that the weights $\phi, \varphi$ and $\psi$ have the scaling property. Suppose, next, that we have the continuous inclusions

$$
\psi^{2} \phi^{2} C_{\phi, \varphi^{2}}^{i, \alpha}(g) \subset \stackrel{\circ}{H_{\phi}^{i}, \psi}(g)
$$


for $i=k, k+1$, with the inclusion norms uniformly bounded for $g$ close to $g_{0}$ in $C_{\phi, 1}^{k+4, \alpha}\left(g_{0}\right)$. Assume finally that $L_{\phi, \psi}(K, g)$ satisfies the weighted elliptic regularity condition, with a uniform constant $C$ in (4.7) for $(K, g)$ close to $\left(K_{0}, g_{0}\right)$ in $\phi^{-1} C_{\phi, 1}^{k+3, \alpha}\left(g_{0}\right) \times C_{\phi, 1}^{k+4, \alpha}\left(g_{0}\right)$. If the source $(\delta J, \delta \rho)$ is in $\psi^{2}\left(\stackrel{\circ}{H}_{\phi, \psi}^{1}(g) \times \stackrel{\circ}{H}_{\phi, \psi}^{0}(g)\right) \cap \psi^{2}\left(C_{\phi, \varphi}^{k+1, \alpha}(g) \times C_{\phi, \varphi}^{k, \alpha}(g)\right)$, with sufficiently small norm, then the solution obtained in Theorem 3.1 with $\varepsilon=0$ is in

$$
\psi^{2}\left(\phi \stackrel{\circ}{H}_{\phi, \psi}^{2}(g) \times \phi^{2} \stackrel{\circ}{H}_{\phi, \psi}^{2}(g)\right) \cap \psi^{2}\left(\phi C_{\phi, \varphi}^{k+2, \alpha}(g) \times \phi^{2} C_{\phi, \varphi}^{k+2, \alpha}(g)\right) .
$$

Proposition 4.4 gives existence of Hölder continuous solutions. We can apply the usual bootstrap arguments to those solutions to obtain smoothness, when all the objects at hand are smooth (compare the proof of Theorem 4.9 below):

Proposition 4.5 (Proposition 3.17 of [12]) Let $k \in \mathbb{N}, \alpha \in(0,1)$, assume that (4.1) with $\ell \geq k+4$ holds, and that (4.2)-(4.4) and (4.6) hold. Suppose moreover that the scaling property holds. Assume that $(K, g) \in C^{k+3, \alpha} \times C^{k+4, \alpha}$ and $(Y, N) \in C_{\phi, \varphi}^{3, \alpha}(g) \times C_{\phi, \varphi}^{4, \alpha}(g)$. If

$\left(\begin{array}{c}J \\ \rho\end{array}\right)\left[(K, g)+\psi^{2} \Phi^{2} P_{(K, g)}^{*}(Y, N)\right]-\left(\begin{array}{c}J \\ \rho\end{array}\right)[(K, g)] \in \psi^{2}\left(C_{\phi, \varphi}^{k+1, \alpha}(g) \times C_{\phi, \varphi}^{k, \alpha}(g)\right)$, then $\left.(Y, N) \in C_{\phi, \varphi}^{k+3, \alpha}(g) \times C_{\phi, \varphi}^{k+4, \alpha}(g)\right)$, thus

$$
(\delta K, \delta g) \in \psi^{2}\left(\phi C_{\phi, \varphi}^{k+2, \alpha}(g) \times \phi^{2} C_{\phi, \varphi}^{k+2, \alpha}(g)\right) .
$$

EXAmple 2.4 (CONTINUED): Applying the last two propositions to the setup of Example 2.4 one finds that smooth solutions of the linearised constraint equations correspond to smooth solutions of the full non-linear constraint equations. This leads then to a Fréchet manifold of smooth solutions near every smooth solution. The argument at the end of proof of Theorem 5.2 justifies the isomorphism property on the overlaps of the coordinate charts, and we have therefore obtained the Fischer-Marsden-Moncrief result:

Theorem 4.6 (Fischer, Marsden, Moncrief [21]) Let $M$ be a compact manifold with boundary. Then the level sets $\mathcal{S}$ of the constraints map form a submanifold of the set of smooth $(K, g)$ 's at all $(K, g)$ which have no KIDs. Each connected component $\mathcal{S}_{0}$ thereof is a Fréchet manifold modeled on $\operatorname{Ker} P_{(K, g)} \subset C^{\infty} \times C^{\infty}$, where $(K, g)$ is an arbitrary element of $\mathcal{S}_{0}$.

Specialising all the considerations so far to the case $K \equiv Y \equiv 0$ one recovers a theorem essentially due to Fischer and Marsden:

Theorem 4.7 (Fischer, Marsden [19]) Let $M$ be a compact manifold with boundary. Then the level sets of the scalar curvature functional on the space of smooth metrics form a submanifold at all $g$ which do not correspond to the space-part of some static solution of the vacuum Einstein equations with a cosmological constant. Each connected component $\mathcal{S}_{0}$ thereof is a Fréchet manifold modeled on the kernel of $D R$, as calculated at some arbitrarily chosen metric $g \in \mathcal{S}_{0}$. 
The argument of the proof of Theorem 3.1 also establishes:

Proposition 4.8 Under the conditions of Proposition 4.4, assume that (3.1) holds, and suppose that $\psi^{-2} P \psi^{2} \Phi^{2} P_{\varepsilon}^{*}$ satisfies the weighted elliptic regularity condition, with the constant $C$ in (4.7) being uniform for $(K, g)$ close to $\left(K_{0}, g_{0}\right)$ in $\phi^{-1} C_{\phi, 1}^{k_{0}+3, \alpha}\left(g_{0}\right) \times C_{\phi, 1}^{k_{0}+4, \alpha}\left(g_{0}\right)$ and $\varepsilon$ small enough. Then Proposition 4.4 remains valid with $k$ replaced by $k_{0}$ and $P^{*}$ replaced by $P_{\varepsilon}^{*}$ when $\varepsilon$ is small enough.

Our first main result is the equivalent of Proposition 4.5 with less regularity conditions on $(K, g)$ :

Theorem 4.9 Let $k \in \mathbb{N}, k \geq 2, \alpha \in(0,1)$, assume that (4.1) with $\ell \geq k+4$ holds, and that (4.2)-(4.4) and (4.6) hold. Under (3.1), suppose that the scaling condition holds for $\varepsilon>0$. Assume that $(K, g) \in C^{k+2, \alpha} \times C^{k+2, \alpha}$ and $(Y, N) \in$ $C_{\phi, \varphi}^{3, \alpha}(g) \times C_{\phi, \varphi}^{4, \alpha}(g)$. For $\varepsilon$ small, if

$$
\left(\begin{array}{c}
J \\
\rho
\end{array}\right)\left[(K, g)+\psi^{2} \Phi^{2} P_{\varepsilon}^{*}(Y, N)\right]-\left(\begin{array}{c}
J \\
\rho
\end{array}\right)[(K, g)] \in \psi^{2}\left(C_{\phi, \varphi}^{k+1, \alpha}(g) \times C_{\phi, \varphi}^{k, \alpha}(g)\right),
$$

then $(Y, N) \in C_{\phi, \varphi}^{k+3, \alpha}\left(g_{\varepsilon}\right) \times C_{\phi, \varphi}^{k+4, \alpha}\left(g_{\varepsilon}\right)$, thus

$$
(\delta K, \delta g) \in \psi^{2}\left(\phi C_{\phi, \varphi}^{k+2, \alpha}(g) \times \phi^{2} C_{\phi, \varphi}^{k+2, \alpha}(g)\right) .
$$

REMARK 4.10 The appearance of $\varepsilon$ in the claim that $(Y, N) \in C_{\phi, \varphi}^{k+3, \alpha}\left(g_{\varepsilon}\right) \times$ $C_{\phi, \varphi}^{k+4, \alpha}\left(g_{\varepsilon}\right)$ is due to the fact that $g$ is a priori not sufficiently differentiable to be able to define spaces such as $C_{\phi, \varphi}^{k+3, \alpha}\left(g_{\varepsilon}\right)$. Any fixed metric uniformly equivalent to $g$, with appropriate weighted differentiability properties, could be used instead of $g_{\epsilon}$.

Proof: It suffices to rewrite the rescaled non-linear elliptic equation (4.9) for $(Y, N)$ as a linear elliptic equation for $(Y, N)$ and freeze coefficients (depending on $(K+\delta K, g+\delta g)$ hence on $(Y, N))$. The interior Hölder estimates [28, Theorem 6.2 .5$, p. 223] on the sets $\hat{\Omega}_{\alpha}$ appearing in the definition of scaling property give the local regularity, and the scaling property gives the global weighted regularity.

\section{Banach manifold structure}

Throughout this section the symbol $g_{0}$ denotes a fixed metric with (local) regularity $C^{m+4, \alpha}$ on $M$.

For $k, l \in\{0, \ldots, m+4\}, \alpha \in(0,1)$ and $g$ a metric in $C^{\max (l, k), \alpha}$, we define the Banach space

$$
\Lambda_{\phi, \psi, \varphi}^{l, k, \alpha}(g)=\stackrel{\circ}{H}_{\phi, \psi}^{l}(g) \cap C_{\phi, \varphi}^{k, \alpha}(g),
$$

equipped with a norm being the sum of the two norms. (It should be clear from (4.10) that this is the topology which one needs to use on the space of 
the metrics when using the methods described above.) When $k \in\{0, \ldots, m+2\}$ and when (4.5) hold, we define the following open subset of $\psi^{2} \phi^{2} \Lambda_{\phi, \psi, \varphi}^{2, k+2, \alpha}\left(g_{0}\right)$ of symmetric two-covariant tensor fields on $M$ :

$\mathcal{A}_{\phi, \psi, \varphi}^{k+2, \alpha}\left(g_{0}\right):=\left\{h \in \psi^{2} \phi^{2} \Lambda_{\phi, \psi, \varphi}^{2, k+2, \alpha}\left(g_{0}\right), g_{0}+h\right.$ is a metric uniformly equivalent to $\left.g_{0}\right\}$.

We note the continuous inclusions:

$$
\mathcal{A}_{\phi, \psi, \varphi}^{k+2, \alpha}\left(g_{0}\right) \subset \psi^{2} \phi^{2} \Lambda_{\phi, \psi, \varphi}^{2, k+2, \alpha}\left(g_{0}\right) \subset C_{\phi, 1}^{k+2, \alpha}\left(g_{0}\right)\left(\subset W_{\phi}^{k+2, \infty}\left(g_{0}\right)\right) .
$$

We have the

Lemma 5.1 Let $k \in\{0, \ldots, m+2\}$ and $l \in\{0, \ldots, k+2\}$. Assume (4.1)-(4.5). Then for all $h \in \mathcal{A}_{\phi, \psi, \varphi}^{k+2, \alpha}\left(g_{0}\right)$ we have

$$
C_{\phi, \varphi}^{l, \alpha}\left(g_{0}+h\right)=C_{\phi, \varphi}^{l, \alpha}\left(g_{0}\right), \quad \stackrel{\circ}{H}_{\phi, \psi}^{l}\left(g_{0}+h\right)=\stackrel{\circ}{H}_{\phi, \psi}^{l}\left(g_{0}\right),
$$

with equivalent norms. In particular,

$$
\mathcal{A}_{\phi, \psi, \varphi}^{k+2, \alpha}\left(g_{0}+h\right)=\mathcal{A}_{\phi, \psi, \varphi}^{k+2, \alpha}\left(g_{0}\right) .
$$

Proof: Let $g=g_{0}+h$, we define $T=\Gamma-\Gamma_{0}$, since $g$ is uniformly equivalent to $g_{0}$ the usual formula for $T$ allows one to estimate this by $C\left|\nabla_{0} h\right|_{g_{0}}$. By the middle inclusion in (5.1) we then have $T \in \phi^{-1} C_{\phi, 1}^{k+1, \alpha}\left(g_{0}\right)$. For a tensor $u$, we have $\|u\|_{g_{0}}$ uniformly equivalent to $\|u\|_{g}$. For the derivatives we write

$$
\nabla u=\nabla_{0} u+\left(\nabla-\nabla_{0}\right) u=\nabla_{0} u-T u .
$$

If $u \in C_{\phi, \varphi}^{l, \alpha}\left(g_{0}\right)$ by the product Lemma 4.2 we obtain $\phi \nabla u \in C_{\phi, \varphi}^{0, \alpha}(g)$. The higher derivatives follow by induction. This shows that $C_{\phi, \varphi}^{l, \alpha}\left(g_{0}\right) \subset C_{\phi, \varphi}^{l, \alpha}(g)$.

We note that the above implies that $-h \in \psi^{2} \phi^{2} C_{\phi, \varphi}^{k+2, \alpha}(g)$, and the reverse inclusion follows by symmetry.

The proof for the Sobolev spaces is identical.

Theorem 5.2 Let $k \in\{2, \ldots, m\}$ and $\alpha \in(0,1)$, and

$$
\operatorname{Ric}\left(g_{0}\right) \in \phi^{-2} C_{\phi, 1}^{k, \alpha}\left(g_{0}\right), \quad K_{0} \in \phi^{-1} C_{\phi, 1}^{k+2, \alpha}\left(g_{0}\right) .
$$

Suppose that the scaling property and the weighted regularity condition hold, and that (4.1)-(4.4) together with (4.6) are satisfied. Assume also that for all 9

$$
(Q, h) \in \psi^{2} \phi \Lambda_{\phi, \psi, \varphi}^{2, k+2, \alpha}\left(g_{0}\right) \times \mathcal{A}_{\phi, \psi, \varphi}^{k+2, \alpha}\left(g_{0}\right)
$$

there exists a compact set $\mathscr{K} \subset M$ such that for all $\stackrel{\circ}{H}_{\phi, \psi}^{1}\left(g_{0}\right)$ vector fields $Y$ and $\stackrel{\circ}{H}_{\phi, \psi}^{2}\left(g_{0}\right)$ functions $N$, both supported in $M \backslash \mathscr{K}$, the inequality (2.12) holds

\footnotetext{
${ }^{9}$ Actually it suffices to assume that this hypothesis holds on $\mathcal{S}_{\left(J_{0}, \rho_{0}\right)}$.
} 
with $\left(K_{0}, g_{0}\right)$ there replaced by $\left(K_{0}+Q, g_{0}+h\right)$. Suppose, further, that for all $(Q, h)$ as in (5.3) the map

$$
(\delta K, \delta g) \rightarrow(J, \rho)\left(K_{0}+Q+\delta K, g_{0}+h+\delta g\right)-(J, \rho)\left(K_{0}+Q, g_{0}+h\right)
$$

is differentiable from a neighborhood of zero in $\psi^{2} \phi \stackrel{\circ}{H}_{\phi, \psi}^{2}\left(g_{0}\right) \times \psi^{2} \phi^{2} \stackrel{\circ}{H}_{\phi, \psi}^{2}\left(g_{0}\right)$ to $\psi^{2} \stackrel{\circ}{H}_{\phi, \psi}^{1}\left(g_{0}\right) \times \psi^{2} \stackrel{\circ}{H}_{\phi, \psi}^{0}\left(g_{0}\right)$. Consider any non-empty connected component of the set of KID-free level-sets:

$$
\begin{aligned}
\mathcal{S}_{\left(J_{0}, \rho_{0}\right)}=\{(Q, h) & \in \psi^{2} \phi \Lambda_{\phi, \psi, \varphi}^{2, k+2, \alpha}\left(g_{0}\right) \times \mathcal{A}_{\phi, \psi, \varphi}^{k+2, \alpha}\left(g_{0}\right), \\
& \left.(J, \rho)\left(K_{0}+Q, g_{0}+h\right)=\left(J_{0}, \rho_{0}\right), \text { Ker } P_{K_{0}+Q, g_{0}+h}^{*}=\{0\}\right\}
\end{aligned}
$$

If there exists a smoothing operation (3.1) with $k_{0}=0$, then $\mathcal{S}_{\left(J_{0}, \rho_{0}\right)}$ is an embedded submanifold of $\psi^{2} \phi \Lambda_{\phi, \psi, \varphi}^{2, k+2, \alpha}\left(g_{0}\right) \times \mathcal{A}_{\phi, \psi, \varphi}^{k+2, \alpha}\left(g_{0}\right)$.

REMARK 5.3 Differentiability of the map (5.4) in weighted Sobolev spaces typically requires $k>n / 2$, this can be actually avoided by requiring instead differentiability of (5.4) as a map from $\psi^{2} \phi \Lambda_{\phi, \psi, \varphi}^{2, k+2, \alpha}\left(g_{0}\right) \times \psi^{2} \phi^{2} \Lambda_{\phi, \psi, \varphi}^{2, k+2, \alpha}\left(g_{0}\right)$ to $\psi^{2} \Lambda_{\phi, \psi, \varphi}^{1, k+1, \alpha}\left(g_{0}\right) \times \psi^{2} \Lambda_{\phi, \psi, \varphi}^{0, k, \alpha}\left(g_{0}\right)$.

REMARK 5.4 We note that a necessary condition for $\mathcal{S}_{\left(J_{0}, \rho_{0}\right)} \neq \emptyset$ is

$$
J_{0} \in \phi^{-2} C_{\phi, 1}^{k+1, \alpha}\left(g_{0}\right), \quad \rho_{0} \in \phi^{-2} C_{\phi, 1}^{k, \alpha}\left(g_{0}\right) .
$$

In any case it seems that the situation of main interest is $J_{0}=\rho_{0}=0$.

REMARK 5.5 The kernel in (5.5) is that of the operator $P_{K_{0}+Q, g_{0}+h}^{*}$ acting from $H_{\phi, \psi}^{1} \times H_{\phi, \psi}^{2}$ to $\phi^{-1} H_{\phi, \psi}^{0} \times \phi^{-2} H_{\phi, \psi}^{0}$. We note that elliptic regularity shows that elements of the kernel are as differentiable as the metric allows, so the elements of the kernel are continuously differentiable solutions satisfying appropriate asymptotic properties.

REMARK 5.6 We do not assume that $\left(J_{0}, \rho_{0}\right)=(J, \rho)\left(K_{0}, \rho_{0}\right)$. Even if this last equality holds, $(0,0)$ will fail to be in $\mathcal{S}$ if there are KIDs at $\left(K_{0}, g_{0}\right)$.

REMARK 5.7 We do not assume $(Q, h)$ to be small.

REMARK 5.8 Some rather general conditions which guarantee existence of smoothing operators (3.1) are given in Appendix B.

Proof: We wish to apply Lemma A.1 with $x=(\delta K, \delta g)$ and

$$
\begin{gathered}
u=D u(0)=\psi^{2} \Phi^{2} P_{\varepsilon,\left(K_{0}+Q_{0}, g_{0}+h_{0}\right)}^{*}, \\
v(x)=\psi^{-2}\left[(J, \rho)\left(K_{0}+Q_{0}+\delta K, g_{0}+h_{0}+\delta g\right)-(J, \rho)\left(K_{0}+Q_{0}, g_{0}+h_{0}\right)\right], \\
D v(0)=\psi^{-2} P_{K_{0}+Q_{0}, g_{0}+h_{0}}, \\
E=\Lambda_{\phi, \psi, \varphi}^{3, k+3, \alpha}\left(g_{0}\right) \times \Lambda_{\phi, \psi, \varphi}^{4, k+4, \alpha}\left(g_{0}\right),
\end{gathered}
$$




$$
\begin{gathered}
F=\psi^{2} \Phi\left(\Lambda_{\phi, \psi, \varphi}^{2, k+2, \alpha}\left(g_{0}\right) \times \Lambda_{\phi, \psi, \varphi}^{2, k+2, \alpha}\right)\left(g_{0}\right), \\
G=\Lambda_{\phi, \psi, \varphi}^{1, k+1, \alpha}\left(g_{0}\right) \times \Lambda_{\phi, \psi, \varphi}^{0, k, \alpha}\left(g_{0}\right),
\end{gathered}
$$

We start by verifying that

$$
L_{\varepsilon}:=\psi^{-2} P_{K_{0}+Q_{0}, g_{0}+h_{0}} \psi^{2} \Phi^{2} P_{\varepsilon,\left(K_{0}+Q_{0}, g_{0}+h_{0}\right)}^{*}
$$

is an isomorphism from $E$ to $G$. We wish to use Theorem 2.2 with $\left(K_{0}, g_{0}\right)$ there replaced with $\left(K_{0}+Q_{0}, g_{0}+h_{0}\right)$, and with $k$ there equal zero; the needed regularity conditions on $\operatorname{Ric}\left(g_{0}+h_{0}\right)$ can be established by the calculations of Lemma 5.1 using (5.2) (recall that $k \geq 2$ ), while the condition on $K_{0}+$ $Q_{0}$ follows immediately from (5.2). The remaining conditions are satisfied by hypothesis. Since there are no KIDs we conclude that $L_{\varepsilon=0} \equiv L_{\phi, \psi}$ (see (2.10)) is an isomorphism from $\stackrel{\circ}{H}_{\phi, \psi}^{3}\left(g_{0}+h_{0}\right) \times \stackrel{\circ}{H}_{\phi, \psi}^{4}\left(g_{0}+h_{0}\right)$ to $\stackrel{\circ}{H}_{\phi, \psi}^{1}\left(g_{0}+h_{0}\right) \times$ $\stackrel{\circ}{H}_{\phi, \psi}^{0}\left(g_{0}+h_{0}\right)$; those spaces coincide with the ones based on $g_{0}$ by Lemma 5.1. The hypothesis of the existence of the smoothing operation (3.1) with $k$ there equal 0 implies that $P_{\varepsilon}^{*} \rightarrow_{\varepsilon \rightarrow 0} P$ in

$$
L\left(\stackrel{\circ}{H}_{\phi, \psi}^{3} \times \stackrel{\circ}{H}_{\phi, \psi}^{4}, \phi^{-1} \stackrel{\circ}{H}_{\phi, \psi}^{2} \times \phi^{-2} \stackrel{\circ}{H}_{\phi, \psi}^{2}\right)
$$

implies that $L_{\varepsilon}$ is an isomorphism from $\stackrel{\circ}{H}_{\phi, \psi}^{3} \times \stackrel{\circ}{H}_{\phi, \psi}^{4}$ to $\stackrel{\circ}{H}_{\phi, \psi}^{1} \times \stackrel{\circ}{H}_{\phi, \psi}^{0}$ for $\varepsilon$ small enough. So $L_{\varepsilon}$ is injective on $E$. The weighted elliptic regularity condition implies Hölder regularity of the solution, and surjectivity follows.

We leave it as an exercise to the reader to prove, using Lemma 4.2 together with the arguments in Lemma 5.1, that:

LEMMA 5.9 Under the conditions of Theorem 5.2, the map (5.4) is smooth from a neighborhood of zero in $\psi^{2} \phi C_{\phi, \varphi}^{k+2, \alpha}\left(g_{0}\right) \times \psi^{2} \phi^{2} C_{\phi, \varphi}^{k+2, \alpha}\left(g_{0}\right)$ to $\psi^{2} C_{\phi, \varphi}^{k+1, \alpha}\left(g_{0}\right) \times$ $\psi^{2} C_{\phi, \varphi}^{k, \alpha}\left(g_{0}\right)$.

This does not suffice to prove differentiability of $v$, because of the topology involved; however, differentiability with respect to the Sobolev topology holds by hypothesis.

It follows that near $\left(Q_{0}, h_{0}\right)$ the set $\mathcal{S}_{\left(J_{0}, \rho_{0}\right)}$ is an embedded submanifold modeled on the kernel of $P_{K_{0}+Q_{0}, g_{0}+h_{0}}$.

REMARK 5.10 The proof above actually gives a foliation of a neighborhood of $\left(Q_{0}, h_{0}\right)$ in $F=\psi^{2} \Phi\left(\Lambda_{\phi, \psi, \varphi}^{2, k+2, \alpha}\left(g_{0}\right) \times \Lambda_{\phi, \psi, \varphi}^{2, k+2, \alpha}\left(g_{0}\right)\right)$. Indeed, under the conditions of Theorem 5.2 we can use Lemma A.2 from Appendix A with the same spaces as those in Theorem 5.2 and with $L=u$. For all $\left(Q_{0}, h_{0}\right) \in \mathcal{S}_{\left(J_{0}, \rho_{0}\right)}$, there is a neighborhood $V$ of zero in $G=\Lambda_{\phi, \psi, \varphi}^{1, k+1, \alpha}\left(g_{0}\right) \times \Lambda_{\phi, \psi, \varphi}^{0, k, \alpha}\left(g_{0}\right)$, such that

$$
\begin{aligned}
& \left\{(Q, h) \in \psi^{2} \phi \Lambda_{\phi, \psi, \varphi}^{2, k+2, \alpha}\left(g_{0}\right) \times \mathcal{A}_{\phi, \psi, \varphi}^{k+2, \alpha}\left(g_{0}\right),\right. \\
& \left.\quad(J, \rho)\left(K_{0}+Q, g_{0}+h\right)=\left(J_{0}, \rho_{0}\right)+(\delta J, \delta \rho), \text { Ker } P_{K_{0}+Q, g_{0}+h}^{*}=\{0\}\right\}_{(\delta J, \delta \rho) \in V}
\end{aligned}
$$


is a foliation. As we can do that for all points $\left(Q_{0}, h_{0}\right) \in \mathcal{S}_{\left(J_{0}, \rho_{0}\right)}$, we obtain a foliation of a neighborhood of $\mathcal{S}_{\left(J_{0}, \rho_{0}\right)}$ in $F$.

In fact, if we denote by $F_{0}$ the open subset of $\psi^{2} \phi \Lambda_{\phi, \psi, \varphi}^{2, k+2, \alpha}\left(g_{0}\right) \times \mathcal{A}_{\phi, \psi, \varphi}^{k+2, \alpha}\left(g_{0}\right)$ of elements $(Q, h)$ such that

$$
\operatorname{ker} P_{K_{0}+Q, g_{0}+h}^{*}=\{0\}
$$

then the map from $F_{0}$ to $G$ defined by:

$$
f(Q, h)=(J, \rho)\left(K_{0}+Q, g_{0}+h\right)-(J, \rho)\left(K_{0}, g_{0}\right),
$$

is a submersion. In particular the levels sets

$$
\left\{(Q, h) \in F_{0}, \quad f(Q, h)=(\delta J, \delta \rho)\right\}_{(\delta J, \delta \rho) \in G},
$$

provide a foliation of $F_{0}$.

For completeness we note the following result:

Corollary 5.11 Every non-empty connected component of $\mathcal{S}_{\left(J_{0}, \rho_{0}\right)}$ defined in (5.5) is a Banach manifold modeled on the kernel of $P_{K_{0}+Q_{0}, g_{0}+h_{0}}$ in $\psi^{2} \Phi\left(\Lambda_{\phi, \psi, \varphi}^{2, k+2, \alpha} \times \Lambda_{\phi, \psi, \varphi}^{2, k+2, \alpha}\right)$ for an (arbitrarily chosen) $\left(Q_{0}, h_{0}\right) \in \mathcal{S}_{\left(J_{0}, \rho_{0}\right)}$.

Proof: An embedded submanifold of a Hausdorff space is necessarily Hausdorff. The local coordinate patches are provided by the maps which model $\mathcal{S}$ on its tangent spaces constructed in the proof of Theorem 5.2. It remains to show that all the kernels are $(Q, h)$-independent. The proof of Theorem 5.2 shows that for all $(Q, h) \in \mathcal{S}_{\left(J_{0}, \rho_{0}\right)}$ there exists a neighborhood thereof in $\mathcal{S}_{\left(J_{0}, \rho_{0}\right)}$ which is arc-connected. It then follows that a connected component of $\mathcal{S}_{\left(J_{0}, \rho_{0}\right)}$ is in fact arc-connected, by observing that the set of metrics in $\mathcal{S}_{\left(J_{0}, \rho_{0}\right)}$ which can be connected to a fixed metric in $\mathcal{S}_{\left(J_{0}, \rho_{0}\right)}$ by a continuous curve contained in $\mathcal{S}_{\left(J_{0}, \rho_{0}\right)}$ is open and closed in $\mathcal{S}_{\left(J_{0}, \rho_{0}\right)}$. Thus for two couples $\left(Q_{0}, h_{0}\right) \in \mathcal{S}_{\left(J_{0}, \rho_{0}\right)}$ and $\left(Q_{p}, h_{p}\right) \in \mathcal{S}_{\left(J_{0}, \rho_{0}\right)}$, there exists an arc $\gamma$ in $\mathcal{S}_{\left(J_{0}, \rho_{0}\right)}$ from one to the other. For each point $x=(Q, h) \in \gamma$, there exists an $r_{x}>0$ such that $\mathcal{S}_{\left(J_{0}, \rho_{0}\right)} \cap B_{F}\left(x, r_{x}\right)$ is diffeomorphic to an open subset of the kernel of $P_{K+Q, g+h}$. As $\gamma$ is compact, there exists a finite number of points $\left\{x_{i}=\left(Q_{i}, h_{i}\right) \in \gamma, i=0, . ., p\right\}$ such that the union of the $B_{F}\left(x_{i}, r_{x_{i}}\right)$ 's covers $\gamma$. This provides a finite chain of diffeomorphisms, a composition of which identifies the kernel of $P_{\left(K+Q_{0}, g+h_{0}\right)}$ with that of $P_{\left(K+Q_{p}, g+h_{p}\right)}$.

\section{Applications}

\subsection{Compact manifolds without boundary}

In this section we apply Theorem 5.2 to the case where $M$ is a compact manifold without boundary. As already pointed out, in this case we take

$$
\phi=\psi=\varphi=1
$$

then the spaces we work with are the standard (non-weighted) Sobolev and Hölder spaces. 
Theorem 6.1 Let $k \geq 2$ and $\alpha \in(0,1)$. Let $g_{0} \in C^{k+2, \alpha}, K_{0} \in C^{k+2, \alpha}$. Then any non-empty connected component of the set

$$
\begin{aligned}
\mathcal{S}_{\left(J_{0}, \rho_{0}\right)}=\{ & (Q, h) \in C^{k+2, \alpha} \times \mathcal{A}_{1,1,1}^{k+2, \alpha}, \\
& \left.(J, \rho)\left(K_{0}+Q, g_{0}+h\right)=\left(J_{0}, \rho_{0}\right), \text { Ker } P_{K_{0}+Q, g_{0}+h}^{*}=\{0\}\right\}
\end{aligned}
$$

is an embedded submanifold of $C^{k+2, \alpha} \times C^{k+2, \alpha}$.

In Theorem 6.1 the kernel of the operator $P_{K_{0}+Q, g_{0}+h}^{*}$ can be taken as that of an operator from $H^{1} \times H^{2}$ to $H^{0} \times H^{0}$, or from $C^{1} \times C^{2}$ to $C^{0} \times C^{0}$, or from $C^{k+1, \alpha} \times C^{k+2, \alpha}$ to $C^{k, \alpha} \times C^{k, \alpha}$.

\subsection{Asymptotically flat manifolds without boundary}

In this section we apply Theorem 5.2 to the case where $M$ is an asymptotically flat manifold without boundary and with compact interior; by definition, this means that $M$ is the union of a compact set with a finite number of regions, called ends, which are diffeomorphic to $\mathbb{R}^{n} \backslash B(0, R)$ for some $R$. We denote by $\hat{\delta}$ some arbitrarily chosen metric of $C^{m+4, \alpha}$ differentiability class which coincides with the Euclidean one in the asymptotically flat regions. Here and in the following sections the index $m$ corresponds to the differentiability of the background, typically $m=\infty$ will be appropriate, however in some situations it might be useful to have a background with finite differentiability. The weight functions are $\phi=r$, with $\varphi$ and $\psi$ - powers of $r$ in the asymptotic regions, which are easily seen to satisfy (4.1)-(4.4) in the asymptotic regions. We extend the function $r$ to a smooth strictly positive function in the compact region, then the requirements on the weight functions are satisfied globally. It is convenient to relabel the $\stackrel{\circ}{H}_{r, r^{\alpha}}^{k}$ and the $C_{r, r^{s}}^{k, \alpha}$ spaces as follows: choose some $m \in \mathbb{N}$, for $k \in\{0, \ldots, m+4\}, \alpha \in(0,1)$ and $\beta \in \mathbb{R}$ we set

$$
\dot{\mathscr{H}}_{k}^{\beta}=\stackrel{\circ}{H}_{r, r^{-n / 2-\beta}}^{k}(\hat{\delta}), C_{k, \alpha}^{\beta}=C_{r, r^{-\beta}}^{k, \alpha}(\hat{\delta}) .
$$

For $\lambda \in \mathbb{R}$ we define

$$
\Lambda_{k, \alpha}^{\lambda}=\Lambda_{r, r^{-n / 2-\lambda}, r^{-\lambda}}^{2, k, \alpha}=\dot{\mathscr{H}}_{2}^{\lambda} \cap C_{k, \alpha}^{\lambda}
$$

We also define for $k \geq 2$ :

$$
\begin{aligned}
& \mathcal{M}_{k, \alpha}=\{g \text { is a metric uniformly equivalent to } \hat{\delta}, \\
& \left.\qquad g-\hat{\delta} \in C_{k, \alpha}^{0},\left|\nabla_{\hat{\delta}}^{(l)}(g-\hat{\delta})\right|_{\hat{\delta}}=o\left(r^{-l}\right), 0 \leq l \leq 2\right\} .
\end{aligned}
$$

For $\gamma \geq 0$ and $k \leq m+2$ we let

$$
\mathcal{A}_{k+2, \alpha}^{-\gamma}=\mathcal{A}_{r, r^{n / 2-2-\gamma}, r^{-\gamma-2+n}}^{2, k+2, \alpha}(\hat{\delta}) \subset C_{k+2, \alpha}^{-\gamma} \subset C_{k+2, \alpha}^{0} .
$$

This corresponds to $\psi=r^{n / 2-2-\gamma}, \varphi=r^{-\gamma-2+n}$, and since $\phi=r$ the condition (4.6) holds precisely for $\gamma \geq 0$. The choice of weights here is justified by Theorem 7.7 in [12]. 
With the labeling above, a metric such that $g-\hat{\delta} \in \mathcal{A}_{k+2, \alpha}^{\beta}$ with $\beta \leq 0$ clearly differs from the Euclidean metric by $O\left(r^{\beta}\right)$. In order to see that this is actually $o\left(r^{\beta}\right)$, for $i \in \mathbb{N}$ set

$$
\Gamma_{i} \equiv \Gamma\left(2^{i} R, 2^{i+1} R\right):=\overline{B\left(0,2^{i+1} R\right)} \overline{B\left(0,2^{i} R\right)} .
$$

Let $f \in \dot{\mathscr{H}}_{0}^{\beta} \cap C_{1}^{\beta}$ and let $x_{n} \in \bar{\Gamma}_{n}$ be any point such that

$$
\left|f\left(x_{n}\right)\right|=\sup _{y \in \Gamma_{n}}|f(y)| .
$$

If $\|f\|_{C_{1}^{\beta}}=0$ there is nothing to prove, otherwise let

$$
r_{n}=\min \left(\frac{1}{8}, \frac{\left|f\left(x_{n}\right)\right| n^{-\beta}}{2\|f\|_{C_{1}^{\beta}}}\right) n .
$$

For $y \in B\left(x_{n}, r_{n}\right)$ we have

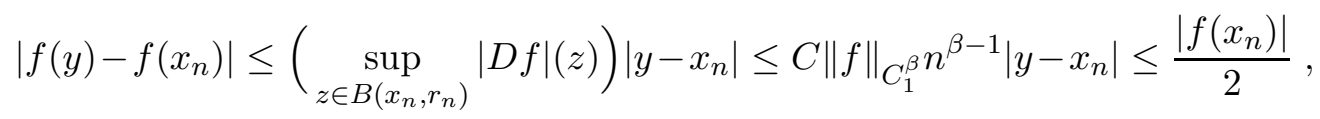

which implies

$$
\int_{B\left(x_{n}, r_{n}\right)} f^{2}(y)|y|^{-2 \beta-n} d^{n} y \geq C^{\prime}\left(\frac{f\left(x_{n}\right)}{2 n^{\beta}}\right)^{2}\left(\frac{r_{n}}{n}\right)^{n} .
$$

The left-hand-side goes to zero as $n$ goes to infinity by the dominated convergence theorem, which easily implies the result.

The reference metric $g_{0}$ will be taken to be such that $g_{0} \in \mathcal{M}_{k+2, \alpha}$. The reference $K_{0}$ can be taken to be zero, but any $K_{0} \in C_{k+2, \alpha}^{-1}$ with $K_{0}=o\left(r^{-1}\right)$ will do; this last condition ensures that the $\mathscr{K}$-set condition of Theorem 5.2 holds, see [12, Section 7] for details.

We take the smoothing operation to be the one in Appendix B.1, the only thing which needs to be checked is the uniform covering condition (B.3): We write

$$
\mathbb{R}^{n} \backslash \overline{B(0, R)}=\cup_{i=0}^{\infty} \Gamma\left(2^{i} R, 2^{i+1} R\right) .
$$

Now $\Gamma(1,2)$ can be covered by a finite number $N$ of ball's $B\left(x_{j},\left|x_{j}\right| / 8\right)$ with $x_{j} \in \Gamma(1,2)$. Then $\Gamma\left(2^{i} R, 2^{i+1} R\right)$ can be covered by $N$ balls $B\left(2^{i} R x_{j}, 2^{i-3} R\left|x_{j}\right|\right)$ with $2^{i} R x_{j} \in \Gamma\left(2^{i} R, 2^{i+1} R\right)$. It is then clear that $\mathbb{R}^{n} \backslash \bar{B}(0, R)$ can be covered by a countable set of balls $B\left(y_{k},\left|y_{k}\right| / 8\right)$ with the property that for all $k \in \mathbb{N}$,

$$
\#\left\{l, B\left(y_{l},\left|y_{l}\right| / 2\right) \cap B\left(y_{k},\left|y_{k}\right| / 2\right) \neq \emptyset\right\} \leq 3 N,
$$

as desired.

We note that the differentiability of the map (5.4) follows from the weighted equivalent of the Schauder ring property of $H^{k} \cap L^{\infty}$. All the remaining hypotheses of Theorem 5.2 will be satisfied by [12] (compare Section 7 there) under the following conditions: 
Theorem 6.2 Let $m \in \mathbb{N}, k \in\{2, \ldots, m\}, \alpha \in(0,1), g_{0} \in \mathcal{M}_{k+2, \alpha}, K_{0} \in C_{k+2, \alpha}^{-1}$ with $K_{0}=o\left(r^{-1}\right)$. Let $\beta \geq 0, \beta \notin\{n-2, n-1\}$. Then any non-empty connected component of the set of KID-free initial data:

$$
\begin{aligned}
\mathcal{S}_{\left(J_{0}, \rho_{0}\right)}=\{ & (Q, h) \in \Lambda_{k+2, \alpha}^{-\beta-1} \times \mathcal{A}_{k+2, \alpha}^{-\beta}, \\
& \left.(J, \rho)\left(K_{0}+Q, g_{0}+h\right)=\left(J_{0}, \rho_{0}\right), \text { Ker } P_{K_{0}+Q, g_{0}+h}^{*}=\{0\}\right\}
\end{aligned}
$$

is an embedded submanifold of $\Lambda_{k+2, \alpha}^{-\beta-1} \times \mathcal{A}_{k+2, \alpha}^{-\beta}$.

In the above the kernel of the operator $P_{K_{0}+Q, g_{0}+h}^{*}$ is viewed as that of an operator from $\dot{\mathscr{H}}_{1}^{\beta+2-n} \times \dot{\mathscr{H}}_{2}^{\beta+2-n}$ to $\dot{\mathscr{H}}_{0}^{\beta-n+1} \times \dot{\mathscr{H}}_{0}^{\beta-n}$. Elliptic regularity implies that elements of this kernel are classically differentiable KIDs such that $Y=o\left(r^{\beta+2-n}\right), N=o\left(r^{\beta+2-n}\right)$. It is known that for $0<\beta<n-2$ there are no non-trivial such KIDs, so we obtain:

Corollary 6.3 Under the conditions of Theorem 6.2, if $\beta \in(0, n-2)$ then all the level sets of the constraints map

$$
\left\{(Q, h) \in \Lambda_{k+2, \alpha}^{-t+1} \times \mathcal{A}_{k+2, \alpha}^{-t+2},(J, \rho)\left(K_{0}+Q, g_{0}+h\right)=\left(J_{0}, \rho_{0}\right)\right\}
$$

are embedded submanifolds of $\Lambda_{k+2, \alpha}^{-t+1} \times \mathcal{A}_{k+2, \alpha}^{-t+2}$.

Thus, within the above set of weights the set of solutions of the vacuum constraint equations does not have any manifold singularities. On the other hand, such singularities will occur at solutions with KIDs if higher values of $\beta$ are used. The interest of such higher $\beta$ 's relies in the fact that the resulting manifolds of solutions possess fixed energy-momentum, or angular momentum, or higher multipoles, depending upon the value of $\beta$.

\subsection{Compact manifold with boundary}

In this section we apply Theorem 5.2 to the case where $\bar{M}$ is a compact manifold with smooth boundary $\partial M$. We wish to construct manifolds of initial data on $\bar{M}$ with prescribed boundary values on $\partial M$, as well as a prescribed number of transverse derivatives at the boundary. Let $\gamma$ be any fixed auxiliary Riemannian metric of $C^{m+4, \alpha}(\bar{M})$ differentiability class. Let $x \geq 0$ be a function that vanishes precisely on $\partial M$, with $d x$ nowhere vanishing there. We start by considering power-law weighted spaces defined as

$$
\begin{gathered}
\stackrel{\circ}{\mathrm{H}}_{k}^{s}=\stackrel{\circ}{H}_{1, x^{-s-n / 2}}^{k}(\gamma), \quad \mathrm{C}_{k, \alpha}^{s}=C_{x, x^{-s}}^{k, \alpha}(\gamma), \\
\Lambda_{k, \alpha}^{s}=\Lambda_{x, x^{-s-n / 2}, x^{-s}}^{2, k, \alpha}=\stackrel{\circ}{\mathrm{H}}_{2}^{s} \cap \mathrm{C}_{k, \alpha}^{s} .
\end{gathered}
$$

We also define for $m+4 \geq k \geq 2$ :

$$
\begin{aligned}
& \mathcal{M}_{k, \alpha}=\{g \text { metric uniformly equivalent to } \gamma, \\
& \left.\qquad g-\gamma \in \mathrm{C}_{k, \alpha}^{0},\left|\nabla_{\gamma}^{(l)}(g-\gamma)\right|_{\gamma}=o\left(x^{-l}\right), 0 \leq l \leq 2\right\} .
\end{aligned}
$$


(this differs from (6.2) by a different background metric and different functional spaces, we hope that this ambiguity will not lead to confusions.) For $\sigma \geq 0$ and $m+2 \geq k \geq 0$ we set

$$
\mathcal{A}_{k+2, \alpha}^{\sigma}=\mathcal{A}_{x, x^{\sigma-2+n / 2}, x^{\sigma-2+n}}^{k+2, \alpha}(\gamma) \subset \mathrm{C}_{k+2, \alpha}^{\sigma} \subset \mathrm{C}_{k+2, \alpha}^{0} .
$$

The first inclusion shows that metrics of the form $g=\gamma+h$ with $h \in$ $\mathcal{A}_{k+2, \alpha}^{\sigma}$ approach $\gamma$ at $\partial M$ at least as $O\left(x^{\sigma}\right)$, and in fact an argument similar to the one in Section 6.2 shows that this is actually at least $o\left(x^{\sigma}\right)$. The above corresponds to $\phi=x, \varphi=x^{\sigma+n-2}, \psi=x^{\sigma+n / 2-2}$, with the choices being justified as follows: In [12, Theorem 5.6] we obtain metrics such that $\delta g$ is in $\stackrel{\circ}{H}_{x, x^{-(s-n+2)-n / 2}} \cap C_{x, x^{-(s-n+2)}}$. It is convenient to number the spaces according to the decay rate of $\delta g$ near the boundary, so we set $\sigma=s-n+2$. In the current set-up we have $\delta g$ in $\psi^{2} \phi^{2}\left(\stackrel{\circ}{H}_{\phi, \psi} \cap C_{\phi, \varphi}\right)$, which after straightforward algebra uniquely leads to the weights above.

In order to obtain the required smoothing operator we use the results in Appendix B.1, we need to justify the covering condition there. Let $T>0$, we set

$$
\left.\mathbb{R}_{+}^{n}=\left\{x=\left(x^{1}, \ldots, x^{n}\right)\right\} \in \mathbb{R}^{n}, x^{n}>0\right\}=\cup_{i=-\infty}^{\infty} \mathcal{B}\left(2^{i} T, 2^{1+i} T\right),
$$

where $\mathcal{B}\left(2^{i} T, 2^{i+1} T\right)=\left\{x=\left(x^{1}, \ldots, x^{n}\right) \in \mathbb{R}^{n}, 2^{i} T<x^{n} \leq 2^{i+1} T\right\}$. We have $\partial \mathbb{R}_{+}^{n}=\left\{x \in \mathbb{R}^{n}, x^{n}=0\right\} . \mathcal{B}(1,2)$ can be covered by closed cubes with edge sizes one, with pairwise intersections empty, or along the faces of the cubes. We choose one of those cubes, call it $K_{1}$, and we cover it by $N(n)$ balls $B\left(x_{i},\left|x_{i}\right| / 8\right)$ with $x_{i} \in K_{1}$; every other cube in $\mathcal{B}(1,2)$ will then be covered by $N(n)$ balls by translating the balls covering $K_{1}$. We cover $\mathcal{B}(1 / 2,1)$ with cubes of edge sizes $1 / 2$, intersecting along the faces only, such that a cube of $\mathcal{B}(1,2)$ intersects precisely $2^{n-1}$ cubes of $\mathcal{B}(1 / 2,1)$. Each cube of $\mathcal{B}(1 / 2,1)$ can be covered by $N(n)$ balls $B\left(x_{i},\left|x_{i}\right| / 8\right)$ with $x_{i}$ in that cube by scaling by $1 / 2$ and translating the balls covering $K_{1}$. An inductive repetition of the procedure leads to a covering of $\mathbb{R}_{+}^{n}$ by a countable set of balls $B\left(y_{k},\left|y_{k}\right| / 8\right)$ with the property that for all $k$,

$$
\#\left\{l, B\left(y_{l},\left|y_{l}\right| / 2\right) \cap B\left(y_{k},\left|y_{k}\right| / 2\right) \neq \emptyset\right\} \leq C_{n} N(n),
$$

for some constant $C_{n}$. Working in local charts, and using partitions of unity, the above construction provides the required covering near the boundary of a manifold.

We refer the reader to [12, Section 5] for a justification of the remaining hypotheses of Theorem 5.2:

Theorem 6.4 Let $k \in\{2, \ldots, m\}, \alpha \in(0,1), g_{0} \in \mathcal{M}_{k+2, \alpha}, K_{0} \in \mathrm{C}_{k+2, \alpha}^{-1}$, with

$$
x\left|K_{0}\right|_{g_{0}}+x^{2}\left|\nabla K_{0}\right|_{g_{0}} \rightarrow_{x \rightarrow 0} 0 .
$$

For $\sigma \geq 0$ when $n>3$ or $\sigma>0$ any non-empty connected component of

$$
\begin{aligned}
\mathcal{S}_{\left(J_{0}, \rho_{0}\right)}=\{ & (Q, h) \in \Lambda_{k+2, \alpha}^{\sigma-1} \times \mathcal{A}_{k+2, \alpha}^{\sigma}, \\
& \left.(J, \rho)\left(K_{0}+Q, g_{0}+h\right)=\left(J_{0}, \rho_{0}\right), \operatorname{Ker} P_{K_{0}+Q, g_{0}+h}^{*}=\{0\}\right\}
\end{aligned}
$$

is an embedded submanifold of $\Lambda_{k+2, \alpha}^{\sigma-1} \times \mathcal{A}_{k+2, \alpha}^{\sigma}$. 
Here the kernel of the operator $P_{K_{0}+Q, g_{0}+h}^{*}$ is, by elliptic regularity, a subspace of $\left(\stackrel{\circ}{\mathrm{H}}_{1}^{-\sigma-n+2} \times \stackrel{\circ}{\mathrm{H}}_{2}^{-\sigma-n+2}\right) \cap\left(C^{k+2}(\bar{M}) \times C^{k+2}(\bar{M})\right)$.

For further reference we note the following result:

Proposition 6.5 Suppose that $\left(K_{0}, g_{0}\right) \in\left(C^{k+2, \alpha} \times C^{k+2, \alpha}\right)(M), k \geq 2, \alpha \in$ $(0,1)$, and let $\Omega \subset M$ be a domain with smooth boundary and compact closure. For all $s \neq(n+1) / 2,(n+3) / 2$, the image of the linearisation $P$, at $\left(K_{0}, g_{0}\right)$, of the constraints map, when defined on $\left(\Lambda_{k+2, \alpha}^{-s+1} \times \Lambda_{k+2, \alpha}^{-s+2}\right)(\Omega)$, is

$$
\left(x^{n-2 s} \mathcal{K}_{0}^{\perp g_{0}}\right) \cap\left(\Lambda_{k+1, \alpha}^{-s} \times \Lambda_{k, \alpha}^{-s}\right) .
$$

Here $\mathcal{K}_{0}$ is the space of KIDs which are in $\stackrel{\circ}{1}_{1}^{s-n} \times \stackrel{\circ}{H}_{2}^{s-n} \subset$ $\left(L^{2} \times L^{2}\right)\left(\Omega, x^{-2 s+n} d \mu_{g_{0}}\right)$, and orthogonality is taken in $\left(L^{2} \times L^{2}\right)\left(\Omega, x^{-2 s+n} d \mu_{g_{0}}\right)$. In other words, the image of $P$ is

$$
\begin{gathered}
\left\{(J, \rho) \in \Lambda_{k+1, \alpha}^{-s} \times \Lambda_{k, \alpha}^{-s} \quad \text { such that }\langle(J, \rho),(Y, N)\rangle_{\left(L^{2} \oplus L^{2}\right)\left(\Omega, d \mu_{g_{0}}\right)}=0\right. \\
\text { for all } \left.(Y, N) \in H_{1}^{s-n} \times H_{2}^{s-n} \text { satisfying } P^{*}(Y, N)=0\right\}
\end{gathered}
$$

Further $P^{-1}(0) \subset \Lambda_{k+2, \alpha}^{-s+1} \times \Lambda_{k+2, \alpha}^{-s+2}$ splits.

Proof: The proof of this result is essentially contained in that of Theorem 6.4; the restriction on the constant $\sigma$ there arises from the requirement that the full non-linear constraint map be well defined, but this restriction is not needed for the linearised problem. We note that a closed space complementing $P^{-1}(0)$ is provided by $\operatorname{Im}\left(\Phi^{2} \psi^{2} P_{\epsilon}^{*}\right)$, for $\epsilon$ small enough (compare the arguments in Appendix A), and that $P$ restricted to this space is an isomorphism onto $\operatorname{Im}(P)$.

Alternative useful weights are the exponential ones, for those we will use Proposition B.5 to obtain the needed regularisation. We take the following weight functions ${ }^{10}$

$$
\phi=x^{2}, \psi=x^{n} e^{-s / x}, \varphi=x^{2 n} e^{-s / x} .
$$

With those choices we note that

$\mathcal{A}_{x^{2}, x^{n} e^{-s / x}, x^{2 n} e^{-s / x}}^{k+2, \alpha} \subset \psi^{2} \phi^{2}\left(H_{\phi, \psi} \cap C_{\phi, \varphi}\right)=\phi^{2}\left(H_{\phi, \psi^{-1}} \cap C_{\phi, \varphi \psi^{-2}}\right)=x^{4}\left(\stackrel{\circ}{H}_{x^{2}, x^{-n} e^{s / x}}^{2} \cap C_{x^{2}, e^{s / x}}^{k+2, \alpha}\right)$.

We define the space

$\mathcal{M}_{k, \alpha}^{e x p}=\{g$ metric uniformly equivalent to $\gamma$,

$$
\left.g-\gamma \in C_{x^{2}, 1}^{k, \alpha},\left|\nabla_{\gamma}^{(l)}(g-\gamma)\right|_{\gamma}=o\left(x^{-2 l}\right), 0 \leq l \leq 2\right\} .
$$

Using the results in Section 5 of [12] one now has:

\footnotetext{
${ }^{10}$ Theorem 5.9 and Proposition 5.10 of [12] provide $\delta g$ in $\psi^{2} \phi^{2}\left(\stackrel{\circ}{H}_{\phi, \psi} \cap C_{\phi, \varphi}\right)=$ $x^{4}\left(\stackrel{\circ}{H}_{x^{2}, x^{-n}} e^{s / x} \cap C_{x^{2}, e^{s / x}}\right)$, which leads to the choices of the weights above.
} 
Theorem 6.6 Let $k \in\{2, \ldots, m\}, \alpha \in(0,1), g_{0} \in \mathcal{M}_{k+2, \alpha}^{e x p}, K_{0} \in x^{-2} C_{x^{2}, 1}^{k+2, \alpha}$, with

$$
x^{2}\left|K_{0}\right|_{g_{0}}+x^{4}\left|\nabla K_{0}\right|_{g_{0}} \rightarrow_{x \rightarrow 0} 0 .
$$

For $s>0$ any non-empty connected component of

$$
\begin{aligned}
& \mathcal{S}_{\left(J_{0}, \rho_{0}\right)}=\left\{(Q, h) \in x^{2} \Lambda_{x^{2}, x^{-n} e^{s / x}, e^{s / x}}^{2, k+2, \alpha} \times \mathcal{A}_{x^{2}, x^{n} e^{-s / x}, x^{2 n} e^{-s / x}}^{k+2,},\right. \\
&\left.(J, \rho)\left(K_{0}+Q, g_{0}+h\right)=\left(J_{0}, \rho_{0}\right), \text { Ker } P_{K_{0}+Q, g_{0}+h}^{*}=\{0\}\right\}
\end{aligned}
$$

is an embedded submanifold of $x^{2} \Lambda_{x^{2}, x^{-n} e^{s / x}, e^{s / x}}^{2, k+2} \times \mathcal{A}_{x^{2}, x^{n} e^{-s / x}, x^{2 n} e^{-s / x}}^{k+2, \alpha}$.

Here the kernel of the operator $P_{K_{0}+Q, g_{0}+h}^{*}$ is a subspace of $\stackrel{\circ}{H}_{x^{2}, x^{n} e^{-s / x}}^{1} \times$ $\stackrel{\circ}{H}_{x^{2}, x^{n} e^{-s / x}}^{2}$; but elliptic regularity shows that elements of the kernel are classically differentiable in the interior, and it is standard to show that they are in $C^{k+2}(\bar{M}) \times C^{k+2}(\bar{M})$.

\subsection{Conformally compactifiable manifolds}

In this section we apply Theorem 5.2 to the case where $M$ is a conformally compactifiable manifold (with a compact conformal boundary at infinity), as in Section 6 of [12]. Let $\gamma$ be $C^{m+4, \alpha}$ conformally compactifiable, so that $\gamma=$ $x^{-2} \bar{\gamma}$, with $x$-defining function for the conformal boundary $\partial M$, and $\bar{\gamma}-$ a Riemannian metric on $\bar{M}$ of $C^{m+4, \alpha}(\bar{M})$ differentiability class. In that context, it is natural to define

$$
\begin{gathered}
\stackrel{\circ}{\mathcal{H}}_{k}^{s}:=\stackrel{\circ}{H}_{1, x^{-s}}^{k}(\gamma)=\stackrel{\circ}{\mathrm{H}}_{k}^{s}(\bar{\gamma}), \\
\mathcal{C}_{k, \alpha}^{s}:=C_{x, x^{-s}}^{k, \alpha}(\bar{\gamma})=\mathrm{C}_{k, \alpha}^{s}(\bar{\gamma}), \\
\Lambda_{k, \alpha}^{s}=\Lambda_{1, x^{-s}, x^{-s}}^{2, k+2, \alpha}(\gamma)=\mathcal{C}_{k, \alpha}^{s} \cap \stackrel{\mathcal{H}}{2}_{2}^{s},
\end{gathered}
$$

and for $t \geq 0$,

$$
\mathcal{A}_{k+2, \alpha}^{t}=\mathcal{A}_{1, x^{t}, x^{t}}^{k+2, \alpha}(\gamma) \subset \mathcal{C}_{k+2, \alpha}^{t} \subset \mathcal{C}_{k+2, \alpha}^{0} .
$$

The space $\mathcal{M}_{k, \alpha}$ is defined similarly to (6.3), but both the background $\gamma$ and the function spaces involved are different now:

$\mathcal{M}_{k, \alpha}=\{g$ metric uniformly equivalent to $\gamma$,

$$
\left.g-\gamma \in \mathcal{C}_{k, \alpha}^{0},\left|\nabla_{\gamma}^{(l)}(g-\gamma)\right|_{\gamma}=o(1), 0 \leq l \leq 2\right\} .
$$

The same locally uniform covering as in the preceding section can be used here, so the smoothing operator of Appendix B.1 applies, leading to:

Theorem 6.7 Let $k \in\{2, \ldots, m\}$ and $\alpha \in(0,1)$. Let $g_{0} \in \mathcal{M}_{k, \alpha}, K_{0}=\lambda_{0} g_{0}+L_{0}$ with $L_{0}, \lambda_{0} \in \mathcal{C}_{k+2, \alpha}^{0},\left|L_{0}\right|_{\gamma} \rightarrow_{x \rightarrow 0} 0,\left|\nabla L_{0}\right|_{\gamma} \rightarrow_{x \rightarrow 0} 0$. Let $t \geq 0, t \notin\{(n-$ $3) / 2,(n-1) / 2,(n+1) / 2\}$. Then any connected component of the set

$$
\begin{aligned}
\mathcal{S}_{\left(J_{0}, \rho_{0}\right)}=\{ & (Q, h) \in \Lambda_{k+2, \alpha}^{t} \times \mathcal{A}_{k+2, \alpha}^{t}, \\
& \left.(J, \rho)\left(K_{0}+Q, g_{0}+h\right)=\left(J_{0}, \rho_{0}\right), \text { Ker } P_{K_{0}+Q, g_{0}+h}^{*}=\{0\}\right\}
\end{aligned}
$$

is a submanifold of $\Lambda_{k+2, \alpha}^{t} \times \mathcal{A}_{k+2, \alpha}^{t}$. For $0 \leq t<(n+1) / 2$ the kernel condition is automatically satisfied. 
Here the kernel of $P_{K_{0}+Q, g_{0}+h}^{*}$ is that for a map from $\mathcal{H}_{1}^{-t} \times \mathcal{H}_{2}^{-t}$ to $\mathcal{H}_{0}^{-t} \times \mathcal{H}_{0}^{-t}$; this can also be reformulated in terms of classical differentiability in appropriately weighted spaces.

Remarks similar to those following Corollary 6.3 concerning the value of $t$ apply here.

REMARK 6.8 For $(Q, h) \in \mathcal{S}_{\left(J_{0}, \rho_{0}\right)}$ we have that $|Q|_{\gamma}=o(1)$ and $|\nabla Q|_{\gamma}=o(1)$.

\section{A Submanifolds, foliations}

The following is a variation of an argument in [17]:

Lemma A.1 Let $E, F$ and $G$ be three Banach spaces, and let $u$ (resp. $v$ ) be a map defined from a neighborhood of 0 in $E$ (resp. F) to $F$ (resp. G) such that $u(0)=0$ (resp. $v(0)=0$ ) and which is differentiable at 0 . We also assume that $D v(0) \circ D u(0)$ is an isomorphism from $E$ to $G$. Then the set $v^{-1}(0)$ is a submanifold of $F$ in a neighborhood of 0.

Proof: For $x \in F$,

$x=\underbrace{D u(0) \circ[D v(0) \circ D u(0)]^{-1} \circ D v(0)(x)}_{\in \operatorname{Im} D u(0)}+\underbrace{x-D u(0) \circ[D v(0) \circ D u(0)]^{-1} \circ D v(0)(x)}_{\in \operatorname{Ker} D v(0)}$.

(It easily follows that $F=\operatorname{Im} D u(0) \oplus \operatorname{Ker} D v(0)$, with both summands closed.) As $D v(0) \circ D u(0)$ is an isomorphism, the inverse function theorem shows that $v \circ u$ is a diffeomorphism in a neighborhood of 0 , so for $x \in F$ close to zero we have

$$
x=\underbrace{u \circ[v \circ u]^{-1} \circ v(x)}_{\in \operatorname{Im} u}+x-u \circ[v \circ u]^{-1} \circ v(x) .
$$

Let us define a map from a neighborhood of zero in $F$ to $F$ by

$$
f(x)=x+u \circ[v \circ u]^{-1} \circ v(x)-D u(0) \circ[D v(0) \circ D u(0)]^{-1} \circ D v(0)(x) .
$$

One clearly has

$$
D f(0)=\mathrm{Id},
$$

and the inverse function theorem shows that $f$ is a diffeomorphism in a neighborhood of zero. We also have

$$
x \in v^{-1}(0) \Longleftrightarrow f(x) \in \operatorname{Ker} D v(0),
$$

(for the " $\Leftarrow "$ part we use the fact that $D v(0) \circ u \circ[v \circ u]^{-1}$ is an isomorphism near zero), so $f$ provides the required map modeling $v^{-1}(0)$ on a linear space.

Lemma A.1 shows how to straighten-up a level set of $v$; one can similarly show existence of foliations by level sets: 
Lemma A.2 Let $E, F$ and $G$ three Banach spaces, $L$ a linear continuous map from $E$ to $F, v$ a map defined from a neighborhood of a point $x_{0}$ in $F$ to $G$, continuously differentiable near $x_{0}$. We assume that $D v\left(x_{0}\right) \circ L$ is an isomorphism from $F$ to $G$. Then there exist a neighborhood $V$ of $y_{0}=v\left(x_{0}\right)$ in $G$ such that the collection of level-sets of $v$,

$$
\{x \in F, v(x)=y\}_{y \in V},
$$

is a foliation of a neighborhood $U$ of $x_{0}$ in $F$.

Proof: We have that $D v(x) \circ L$ is a diffeomorphism for $x \in F$ close to $x_{0}$, one then has as in Lemma A.1 that

$$
F=\operatorname{Im} L \oplus \operatorname{Ker} D v(x),
$$

and one easily checks that $\operatorname{Im} L$ is closed (recall that all maps are continuous). So $D v(x)$ is surjective and its kernel splits. From [25, p. 21] the map $v$ is then a submersion near $x_{0}$. In particular, again from [25, p. 20], there exist $U$ neighborhood of $x_{0}$ in $F, V$ neighborhood of $y_{0}$ in $G$ and two isomorphisms $\varphi: U \longrightarrow U_{1} \times U_{2}$ ( $U_{1}$ and $U_{2}$ open subset of some Banach spaces) and $\psi$ : $V \longrightarrow V_{2}\left(V_{2}\right.$ open subset of some Banach spaces with $\left.U_{2} \subset V_{2}\right)$ such that

$$
\psi \circ v \circ \varphi^{-1}: U_{1} \times U_{2} \longrightarrow V_{2},
$$

is the projection on the second axis. This gives the desired foliation of $U$.

\section{B Two weighted smoothing operators}

In this appendix we will show how to define smoothing operators as needed in the body of the paper; this will require a set of conditions on the functions $\phi$ and $\varphi$, compatible with the usual settings of interest in general relativity. The technique of Appendix B.2 seems to be somewhat simpler than that of Appendix B.1, and does not require any covering conditions. However, covering conditions arise naturally when regularising functions in weighted Sobolev classes, therefore it seemed of interest to us to present both methods.

\section{B.1 Smoothing with locally uniformly finite coverings}

Throughout this appendix we assume that the manifold $M$ is an open subset of $\mathbb{R}^{n}$, equipped with an Euclidean metric (which is of course not the physical space metric we are interested in), and we will be regularising functions. The

regularisation can then be applied to tensor fields on more general manifolds by using coordinate patches, partitions of unity, and usual covering arguments. 
We assume that $\phi$ and $\varphi$ verify (4.1)-(4.3). For all $p \in M$, we denote by $B_{p}$, the open ball of center $p$ with radius $\phi(p) / 2$. We require that ${ }^{11}$ for all $p \in M$,

$$
B(p, \phi(p)) \subset M .
$$

For $\rho \in[1, \infty)$ we shall use the following notation:

$$
B_{i}^{\rho}=B\left(p_{i}, \phi\left(p_{i}\right) / \rho\right)
$$

Our next restriction is that the manifold can be covered by a countable collection of balls $B_{i}^{8}$,

$$
M=\cup_{i=1}^{\infty} B_{i}^{8},
$$

such that there exists an $N \in \mathbb{N}$ so that for all $i \in \mathbb{N}$,

$$
\#\left\{j, B_{i}^{2} \cap B_{j}^{2} \neq \emptyset\right\} \leq N .
$$

For $p \in M$, we set

$$
\varphi_{p}: B(0,1 / 2) \ni z \mapsto p+\phi(p) z \in B_{p} .
$$

This implies that for all functions $u$ on $M$ and all multi-indices $\gamma$ we have

$$
\partial_{z}^{\gamma}\left(u \circ \varphi_{p}\right)=\phi(p)^{|\gamma|}\left(\partial^{\gamma} u\right) \circ \varphi_{p} .
$$

Using (4.2) and (4.3) it is easy to see that we have:

Lemma B.1 For $\rho \in[2,8]$ the following norms on $C_{\phi, \varphi}^{k, \alpha}(M)$ are equivalent:

$$
\begin{aligned}
\|u\|_{C_{\phi, \varphi}^{k, \alpha}(M)} & \sim \sup _{i \in \mathbb{N}}\|u\|_{C_{\phi, \varphi}^{k, \alpha}\left(B_{i}^{\rho}\right)} \\
& \sim \sup _{i \in \mathbb{N}}\|u\|_{C_{\phi\left(p_{i}\right), \varphi\left(p_{i}\right)}^{k, \alpha}\left(B_{i}^{\rho}\right)} \\
& \sim \sup _{i \in \mathbb{N}}\left\|u \circ \varphi_{p_{i}}\right\|_{C_{1, \varphi}^{k, \alpha}\left(p_{i}\right)}(B(0,1 / \rho))
\end{aligned}
$$

We now construct a convenient partition of unity:

Lemma B.2 There exists a partition of unity

$$
\sum_{i=1}^{\infty} \zeta_{i}=1
$$

with smooth functions $\zeta_{i} \geq 0$, and $\zeta_{i}=0$ outside $B_{i}^{4}$, such that for all $l \in \mathbb{N}$ and $\alpha \in(0,1)$ there exists a constant $C(l, \alpha)$ so that for all $i \in \mathbb{N}$,

$$
\left\|\zeta_{i}\right\|_{C_{\phi, 1}^{l, \alpha}(M)} \leq C(l, \alpha)
$$

\footnotetext{
${ }^{11}$ Equation (B.1) can be replaced by the weaker condition that there exists $\mu>0$ such that for all $p \in M$ we have $B(p, \mu \phi(p)) \subset M$, as changing $\phi$ to $\mu \phi$ for a positive constant $\mu$ leads to equivalent norms. So, e.g. in the asymptotically flat case, one actually has to replace the weight $\phi=r$ for $r \geq R$ by $\phi=r / 2 R$. Any such rescaling lead to obvious changes in the hypotheses needed for the covering arguments below.
} 
ProOF: Let $\chi$ be a smooth non-negative function on $\mathbb{R}^{n}$ such that $\chi=1$ on $B(0,1 / 8)$ and $\chi=0$ outside $B(0,1 / 4)$. We define

$$
\chi_{i}:=\chi \circ \varphi_{p_{i}}^{-1}, \quad \zeta_{i}:=\frac{\chi_{i}}{\sum_{j=1}^{\infty} \chi_{j}} .
$$

Let us show that the sum in the definition above is well defined and greater than one. If $x \in B_{i}^{4}$, there exists at most $N$ balls $B_{j}^{4}$, with $N$ given by (B.3), such that $x \in B_{j}^{4}$. Since $\chi_{j}$ has support in $B_{j}^{4}$, the sum at $x$ is over a finite set. If $x \in B_{i}^{8}$ then $\chi_{i}(x)=1$ thus the sum is not less than one. If $x \in B_{i}^{4} \backslash B_{i}^{8}$, from (B.2), $x$ must be in some $B_{j}^{8}$ thus $\chi_{j}(x)=1$ and then, again, the sum is greater than or equal to one.

Now, $\chi_{i}$ has compact support so is in $C_{\phi, 1}^{l, \alpha}(M)$, and from Lemma B.1

$\left\|\chi_{i}\right\|_{C_{\phi, 1}^{l, \alpha}(M)}=\left\|\chi_{i}\right\|_{C_{\phi, 1}^{l, \alpha}\left(B_{i}^{4}\right)} \leq C^{\prime}\left\|\chi_{i} \circ \varphi_{p_{i}}\right\|_{C_{1,1}^{l, \alpha}(B(0,1 / 4))}=C^{\prime}\|\chi\|_{C_{1,1}^{l, \alpha}(B(0,1 / 4))}=: C_{1}$,

where $C_{1}$ depends upon $l$ and $\alpha$ but does not depend upon $i$. So we have that

$$
\left\|\sum_{j=1}^{\infty} \chi_{j}\right\|_{C_{\phi, 1}^{l, \alpha}\left(B_{i}^{2}\right)} \leq \sum_{\left\{j, B_{i}^{2} \cap B_{j}^{2} \neq \emptyset\right\}}\left\|\chi_{j}\right\|_{C_{\phi, 1}^{l, \alpha}(M)} \leq N C_{1} .
$$

and thus

$$
\left\|\sum_{j=1}^{\infty} \chi_{j}\right\|_{C_{\phi, 1}^{l, \alpha}(M)} \leq N C_{1} .
$$

Finally, as the sum is greater than or equal to one it is easy to see that the $\zeta_{i}$ 's satisfy the desired properties.

Let $\theta$ by any smooth strictly positive function on $\mathbb{R}^{n}$, with support in $B(0,1)$ and such that

$$
\int_{\mathbb{R}^{n}} \theta=1
$$

For $\varepsilon>0$ we set

$$
\theta_{\varepsilon}(x)=\frac{1}{\varepsilon^{n}} \theta\left(\frac{x}{\varepsilon}\right) .
$$

For $u$ a function on $M$ and $\varepsilon>0$, we define

$$
\begin{gathered}
u_{i}=\zeta_{i} u, \quad \hat{u}_{i}=u_{i} \circ \varphi_{p_{i}}, \\
\hat{u}_{i, \varepsilon}=\theta_{\varepsilon} * \hat{u}_{i}, \quad u_{i, \varepsilon}=\hat{u}_{i, \varepsilon} \circ \varphi_{p_{i}}^{-1}, \quad u_{\varepsilon}=\sum_{i=1}^{\infty} u_{i, \varepsilon}
\end{gathered}
$$

Proposition B.3 Let $u \in C_{\phi, \varphi}^{k, \alpha}(M)$. For all $\varepsilon \in(0,1 / 4)$ and all $m \in \mathbb{N}$ we have $u_{\varepsilon} \in C_{\phi, \varphi}^{m}(M)$. Further, $u_{\varepsilon}$ converges to $u$ in $C_{\phi, \varphi}^{k, \alpha}(M)$ as $\varepsilon$ goes to zero.

Proof: First remark that as the $u_{i}$ 's have support in $B_{i}^{4}$, then for $\varepsilon<1 / 4$ the functions $u_{i, \varepsilon}$ have support in $B_{i}^{2}$. It follows that on $B_{i}^{2}$ we have

$$
u_{\varepsilon}=\sum_{\left\{j, B_{i}^{2} \cap B_{j}^{2} \neq \emptyset\right\}} u_{j, \varepsilon} .
$$


Lemma B.1 and B.2 together with standard properties of convolution in $\mathbb{R}^{n}$ imply

$$
\begin{aligned}
\left\|u_{\varepsilon}\right\|_{C_{\phi, \varphi}^{k, \alpha}\left(B_{i}^{2}\right)} & \leq \sum_{\left\{j, B_{i}^{2} \cap B_{j}^{2} \neq \emptyset\right\}}\left\|u_{j, \varepsilon}\right\|_{C_{\phi, \varphi}^{k, \alpha}\left(B_{i}^{2}\right)} \\
& =\sum_{\left\{j, B_{i}^{2} \cap B_{j}^{2} \neq \emptyset\right\}}\left\|u_{j, \varepsilon}\right\|_{C_{\phi, \varphi}^{k, \alpha}\left(B_{i}^{2} \cap B_{j}^{2}\right)} \\
& \leq \sum_{\left\{j, B_{i}^{2} \cap B_{j}^{2} \neq \emptyset\right\}}\left\|u_{j, \varepsilon}\right\|_{C_{\phi, \varphi}^{k, \alpha}\left(B_{j}^{2}\right)} \\
& \leq C \sum_{\left\{j, B_{i}^{2} \cap B_{j}^{2} \neq \emptyset\right\}}\left\|\hat{u}_{j, \varepsilon}\right\|_{C_{\phi\left(p_{j}\right), \varphi\left(p_{j}\right)}^{k, \alpha}(B(0,1 / 2))} \\
& \leq C \sum_{\left\{j, B_{i}^{2} \cap B_{j}^{2} \neq \emptyset\right\}}\left\|\hat{u}_{j}\right\|_{C_{\phi\left(p_{j}\right), \varphi\left(p_{j}\right)}^{k, \alpha}(B(0,1 / 2))} \\
& \leq C^{\prime} \sum_{\left\{j, B_{i}^{2} \cap B_{j}^{2} \neq \emptyset\right\}}\left\|u_{j}\right\|_{C_{\phi, \varphi}^{k, \alpha}\left(B_{i}^{2}\right)} \\
& \leq N C^{\prime \prime} C(k, \alpha)\|u\|_{C_{\phi, \varphi}^{k, \alpha}(M)}
\end{aligned}
$$

In particular $u_{\varepsilon} \in C_{\phi, \varphi}^{k, \alpha}(M)$, with norm uniformly bounded in $\varepsilon$. Let us now show that in fact $u_{\varepsilon}$ is also in $C_{\phi, \varphi}^{k+l}(M)$ for any $l \geq 0$. First, we have:

$$
\begin{aligned}
\sup _{B_{i}^{2}}\left|\varphi \phi^{k+l} \partial^{k+l} u_{i, \varepsilon}\right| & \leq C \sup _{B_{i}^{2}}\left|\varphi\left(p_{i}\right) \phi^{k+l}\left(p_{i}\right) \partial^{k+l} u_{i, \varepsilon}\right| \\
& =C \sup _{B(0,1 / 2)}\left|\varphi\left(p_{i}\right) \partial^{k+l} \hat{u}_{i, \varepsilon}\right| \\
& \leq C\left\|\partial^{l} \theta_{\varepsilon}\right\|_{L^{1}(B(0,1 / 2))}\left\|\varphi\left(p_{i}\right) \partial^{k} \hat{u}_{i}\right\|_{L^{\infty}(B(0,1 / 2))} \\
& =C \frac{1}{\varepsilon^{l}}\left\|\partial^{l} \theta\right\|_{L^{1}\left(\mathbb{R}^{n}\right)}\left\|\varphi\left(p_{i}\right) \partial^{k} \hat{u}_{i}\right\|_{L^{\infty}(B(0,1 / 2))} \\
& \leq C^{\prime} \frac{C_{l}}{\varepsilon^{l}}\left\|\varphi \phi^{k} \partial^{k} u_{i}\right\|_{L^{\infty}\left(B_{i}^{2}\right)} \\
& \leq C^{\prime \prime} \frac{C_{l}}{\varepsilon^{l}}\|u\|_{C_{\phi, \varphi}^{k}(M)} .
\end{aligned}
$$

Thus we have

$$
\sup _{B_{i}^{2}}\left|\varphi \phi^{k+l} \partial^{k+l} u_{\varepsilon}\right| \leq \sum_{\left\{j, B_{i}^{2} \cap B_{j}^{2} \neq \emptyset\right\}} \sup _{B_{i}^{2} \cap B_{j}^{2}}\left|\varphi \phi^{k+l} \partial^{k+l} u_{j, \varepsilon}\right| \leq N C^{\prime \prime \prime} \frac{C_{l}}{\varepsilon^{l}}\|u\|_{C_{\phi, \varphi}^{k}(M)},
$$

and then, using Lemma B.1,

$$
\sup _{M}\left|\varphi \phi^{k+l} \partial^{k+l} u_{\varepsilon}\right| \leq N C^{\prime \prime \prime} \frac{C_{l}}{\varepsilon^{l}}\|u\|_{C_{\phi, \varphi}^{k}(M)}
$$

which gives $u_{\varepsilon} \in C_{\phi, \varphi}^{k+l}(M)$ for any $l \geq 0$.

Let us pass now to the proof that $u_{\varepsilon}$ converges to $u$ in $C_{\phi, \varphi}^{k, \alpha}(M)$. We have

$$
\begin{aligned}
\left\|u_{i, \varepsilon}-u_{i}\right\|_{C_{\phi, \varphi}^{k, \alpha}\left(B_{i}^{2}\right)} & \leq C\left\|\hat{u}_{i, \varepsilon}-\hat{u}_{i}\right\|_{C_{1, \varphi\left(p_{i}\right)}^{k, \alpha}}(B(0,1 / 2)) \\
& \leq C^{\prime} \varepsilon^{\alpha}\left\|\hat{u}_{i}\right\|_{C_{1, \varphi\left(p_{i}\right)}^{k, \alpha}(B(0,1 / 2))} \\
& \leq C^{\prime \prime} \varepsilon^{\alpha}\|u\|_{C_{\phi, \varphi}^{k, \alpha}(M)}
\end{aligned}
$$

Thus as $u_{\varepsilon}-u=\sum_{i=1}^{\infty}\left(u_{i, \varepsilon}-u_{i}\right)$, on $B_{i}^{2}$, we have

$$
\left\|u_{\varepsilon}-u\right\|_{C_{\phi, \varphi}^{k, \alpha}\left(B_{i}^{2}\right)} \leq \sum_{\left\{j, B_{i}^{2} \cap B_{j}^{2} \neq 0\right\}}\left\|u_{j, \varepsilon}-u_{j}\right\|_{C_{\phi, \varphi}^{k, \alpha}\left(B_{i}^{2} \cap B_{j}^{2}\right)} \leq N C^{\prime \prime} \varepsilon^{\alpha}\|u\|_{C_{\phi, \varphi}^{k, \alpha}\left(B_{i}^{2}\right)},
$$

then finally

$$
\left\|u_{\varepsilon}-u\right\|_{C_{\phi, \varphi}^{k, \alpha}(M)} \leq N C^{\prime} \varepsilon^{\alpha}\|u\|_{C_{\phi, \varphi}^{k, \alpha}(M)} .
$$




\section{B.2 Smoothing using a weighted convolution}

For the exponential weights considered in Section 6.3 the uniform covering condition of the previous section seems to be awkward to verify directly, if true. It is therefore convenient to proceed differently. In what follows we will assume that $M$ is an open domain in $\mathbb{R}^{n}$, this can again be gotten rid of by using partitions of unity and passing to local charts. As in the preceding section, we assume that $\phi$ and $\varphi$ satisfy (4.1)-(4.3) and that (B.1) holds.

LEMma B.4 The following norms on $C_{\phi, \varphi}^{k, \alpha}(M)$ are equivalent:

$$
\begin{aligned}
\|u\|_{C_{\phi, \varphi}^{k, \alpha}(M)} & \sim \sup _{p \in M}\|u\|_{C_{\phi, \varphi}^{k, \alpha}\left(B_{p}\right)} \\
& \sim \sup _{p \in M}\|u\|_{C_{\phi(p), \varphi(p)}^{k, \alpha}\left(B_{p}\right)} .
\end{aligned}
$$

For $u \in C_{\phi, \varphi}^{k, \alpha}(M)$ and for $\epsilon \in(0,1 / 2)$, we define the smooth function on $M$ (here $\theta$ is as in the preceding section):

$$
\begin{aligned}
\widetilde{u}_{\varepsilon}(x) & =\int_{\mathbb{R}^{n}} \frac{1}{\varepsilon^{n} \phi(x)^{n}} \theta\left(\frac{x-y}{\varepsilon \phi(x)}\right) u(y) d^{n} y \\
& =\int_{y \in B(x, \varepsilon \phi(x))} \frac{1}{\varepsilon^{n} \phi(x)^{n}} \theta\left(\frac{x-y}{\varepsilon \phi(x)}\right) u(y) d^{n} y \\
& =\int_{z \in B(0,1)} \theta(z) u(x-\varepsilon \phi(x) z) d^{n} z .
\end{aligned}
$$

Proposition B.5 Let $u \in C_{\phi, \varphi}^{k, \alpha}(M)$. For all $\varepsilon \in(0,1 / 2)$ and all $m \in \mathbb{N}$ we have $\widetilde{u}_{\varepsilon} \in C_{\phi, \varphi}^{m}(M)$. Further, $\widetilde{u}_{\varepsilon}$ converges to $u$ in $C_{\phi, \varphi}^{k, \alpha}(M)$ as $\varepsilon$ goes to zero.

Proof: We first show that $\widetilde{u}_{\varepsilon} \in C_{\phi, \varphi}^{k, \alpha}(M)$ with norm bounded independently of $\varepsilon$. We have

$$
\left|\varphi(x) \widetilde{u}_{\varepsilon}(x)\right| \leq \sup _{y \in B^{n}(x, \varepsilon \phi(x))}|\varphi(x) u(y)| \leq C\|u\|_{C_{\phi, \varphi}^{k, \alpha}(M)},
$$

where the last inequality comes from Lemma B.4. For the first derivatives, we have

$$
\partial_{i} \widetilde{u}_{\varepsilon}(x)=\int_{z \in B^{n}(0,1)} \theta(z) \partial_{j} u(x-\varepsilon \phi(x) z)\left(\delta_{i}^{j}-\varepsilon \partial_{i} \phi(x) z^{j}\right) d^{n} z .
$$

So from equation (2.5) and Lemma B.4 we have

$$
\left|\varphi(x) \phi(x) \partial_{i} \widetilde{u}_{\varepsilon}(x)\right| \leq C \sup _{y \in B^{n}(x, \varepsilon \phi(x))}|\varphi(x) \phi(x) \partial u(y)| \leq C^{\prime}\|u\|_{C_{\phi, \varphi}^{k, \alpha}(M)} .
$$

It should be clear that similar inequalities are true for the $k^{\text {th }}$ derivatives and for the Hölder quotients, leading to

$$
\left\|\widetilde{u}_{\varepsilon}\right\|_{C_{\phi, \varphi}^{k, \alpha}(M)} \leq C\|u\|_{C_{\phi, \varphi}^{k, \alpha}(M)} .
$$


We leave it as an exercise to the reader to show in a manner similar to that in the preceding section that $\widetilde{u}_{\varepsilon}$ is in fact in $C_{\phi, \varphi}^{k+l}(M)$ for all $l \in \mathbb{N}$.

Writing

$$
\widetilde{u}_{\varepsilon}(x)-u(x)=\int_{z \in B^{n}(0,1)} \theta(z)[u(x-\varepsilon \phi(x) z)-u(x)] d^{n} z,
$$

one similarly shows that

$$
\left\|\widetilde{u}_{\varepsilon}-u\right\|_{C_{\phi, \varphi}^{k, \alpha}(M)} \leq C\|u\|_{C_{\phi, \varphi}^{k, \alpha}(M)} \varepsilon^{\alpha}
$$

so that $\widetilde{u}_{\varepsilon}$ converges to $u$ in $C_{\phi, \varphi}^{k, \alpha}(M)$ when $\varepsilon$ goes to zero, as required.

ACKNowledgements: PTC acknowledges useful discussions with L. Andersson and R. Bartnik.

\section{References}

[1] L. Andersson, Momenta and reduction in general relativity, Jour. Geom. Phys. 4 (1987), 289-314.

[2] — Elliptic systems on manifolds with asymptotically negative curvature, Indiana Univ. Math. Jour. 42 (1993), 1359-1388.

[3] L. Andersson and P.T. Chruściel, On asymptotic behavior of solutions of the constraint equations in general relativity with "hyperboloidal boundary conditions", Dissert. Math. 355 (1996), 1-100.

[4] T. Aubin, Espaces de Sobolev sur les variétés Riemanniennes, Bull. Sci. Math., II. Ser. 100 (1976), 149-173.

[5] — Nonlinear analysis on manifolds. Monge-Ampère equations, Springer, New York, Heidelberg, Berlin, 1982.

[6] R. Bartnik, The mass of an asymptotically flat manifold, Comm. Pure Appl. Math. 39 (1986), 661-693.

[7] R. Bartnik, New definition of quasilocal mass, Phys. Rev. Lett. 62 (1989), 2346-2348.

[8] _ Phase space for the Einstein equations, unpublished (1996), http: //www.ise.canberra.edu.au/mathstat/StaffPages/Robert2.htm.

[9] R. Beig and P.T. Chruściel, Killing Initial Data, Class. Quantum. Grav. 14 (1996), A83-A92, A special issue in honour of Andrzej Trautman on the occasion of his 64th Birthday, J.Tafel, editor.

[10] D. Brill, S. Deser, and L. Fadeev, Sign of gravitational energy, Phys. Lett. 26A (1968), 538-539. 
[11] D. Christodoulou and Y. Choquet-Bruhat, Elliptic systems in $H_{s, \delta}$ spaces on manifolds which are Euclidean at infinity, Acta. Math. 146 (1981), 129-150.

[12] P.T. Chruściel and E. Delay, On mapping properties of the general relativistic constraints operator in weighted function spaces, with applications, Mém. Soc. Math. de France. (2003), in press, gr-qc/0301073.

[13] J. Corvino, Scalar curvature deformation and a gluing construction for the Einstein constraint equations, Commun. Math. Phys. 214 (2000), 137-189.

[14] , lecture in Oberwolfach, July 2000.

[15] J. Corvino and R. Schoen, On the asymptotics for the vacuum Einstein constraint equations, gr-qc/0301071, 2003.

[16] _ Vacuum spacetimes which are identically Schwarzschild near spatial infinity, talk given at the Santa Barbara Conference on Strong Gravitational Fields, June 22-26, 1999, http://doug-pc.itp.ucsb.edu/online/ gravity_c99/schoen/.

[17] E. Delay, Étude locale d'opérateurs de courbure sur l'espace hyperbolique, Jour. Math. Pures Appl. (9) 78 (1999), 389-430.

[18] J. Eells, Jr., A setting for global analysis, Bull. Amer. Math. Soc. 72 (1966), 751-807.

[19] A.E. Fischer and J.E. Marsden, Deformations of the scalar curvature, Duke Math. Jour. 42 (1975), 519-547.

[20] _ The initial value problem and the dynamical formulation of general relativity, Einstein Centenary Volume (Hawking and Israel, eds.), Cambridge University Press, Cambridge, 1979, pp. 138-211.

[21] A.E. Fischer, J.E. Marsden, and V. Moncrief, The structure of the space of solutions of Einstein's equations I: One Killing field, Ann. Inst. H. Poincaré 23 (1980), 147-194.

[22] C.R. Graham and J.M. Lee, Einstein metrics with prescribed conformal infinity on the ball, Adv. Math. 87 (1991), 186-225.

[23] E. Hebey, Sobolev spaces on Riemannian manifolds, Lecture Notes in Mathematics, vol. 1635. Berlin: Springer, 116 p., 1996.

[24] A. Kriegl and P.W. Michor, The convenient setting of global analysis, Mathematical Surveys and Monographs, vol. 53, American Mathematical Society, Providence, RI, 1997.

[25] S. Lang, Introduction to differentiable manifolds, second ed., Universitext, Springer-Verlag, New York, 2002.

[26] J.M. Lee, Fredholm operators and Einstein metrics on conformally compact manifolds, math.DG/0105046, 2001. 
[27] V. Moncrief, Spacetime symmetries and linearization stability of the Einstein equations. I, Jour. Math. Phys. 16 (1975), 493-498.

[28] C.B. Morrey, Multiple integrals in the calculus of variation, Springer Verlag, Berlin, Heidelberg, New York, 1966.

[29] R.S. Palais, Foundations of global non-linear analysis, W. A. Benjamin, Inc., New York-Amsterdam, 1968. 